\title{
Dynamic behaviours of water droplets impacting on laser ablated surfaces
}

Yukui Cai, Xichun Luo* Yankang Tian, Rashed Md. Murad Hasan, Wenlong Chang, Yi Qin

Centre for Precision Manufacturing, DMEM, University of Strathclyde, UK

\begin{abstract}
In order to reveal the underlying mechanism of surface microstructure-determined wetting states, this paper adopted Volume of Fluid (VOF) method to investigate the dynamic behaviours of water droplets impacting on surfaces with different structures at low and high Weber numbers. The simulation results showed that the high and stable pressure of air pockets is critical for the formation of the superhydrophobicity. A superhydrophobic substrate will result in shorter recoiling time and longer rebound time for water droplet than the hydrophobic substrate. Furthermore, superhydrophobic surface resulted in higher kinetic energy for water droplet than hydrophobic surfaces, which is the underlying mechanism of microstructureenabled self-cleaning function. High-speed camera tests of laser processed surface microstructures were conducted to validate the observation in dynamic impacting simulation. The results in both high-speed camera testing and VOF simulation proved that water droplet will have a lower adhesion force when impacting superhydrophobic surface than hydrophobic surface.
\end{abstract}

Keywords: Superhydrophobic; VOF simulation; contact angle; high-speed camera

\section{Introduction}

A superhydrophobic surface is defined as a surface with a water contact angle (CA) larger than $150^{\circ}$ and a sliding angle lower than $10^{\circ}$ [1-3]. In recent years, the superhydrophobic phenomenon has drawn a lot of attention for its useful functions such as self-cleaning, corrosion resistance, anti-icing, drag reduction, heat transfer and anti-bacteria [4-9]. Recently, considerable research efforts have been made to create superhydrophobic surfaces through either surface coating by using electrochemical deposition $[8,10,11]$, laser plasma-type atom beam [12], chemical vapour deposition [13], sol-gel processing [14], electrospinning [15], solution immersion $[16,17]$ or surface structuring by using wire electrical discharge machining [18], wet chemical reaction [19], lithography [20], micro milling [21], laser ablation [22] etc. Thus, a tremendous amount of research focused on the manufacturing process of 
superhydrophobic surfaces but paying little attention to the dynamic impacting process of water droplet on the superhydrophobic surfaces.

Revealing the dynamic behaviour of the water droplet impacting on the superhydrophobic surface is critical to understand the underlying mechanism of hydrophobicity and directly influences their functions. Modern technologies for visualising shape, such as particle image velocimetry (PIV), planar laser induced fluorescence (PLIF), thermal imager and high-speed camera allow deeper understanding of the shape variation, velocity field, heat and mass transfer of a sessile droplet [23,24]. For instance, the high-speed cameras are normally used to analyse the dynamic behaviour of water droplet on different substrates through properties like watersolid contact time, droplet morphology and bounce height etc. However, it is challenging to clearly observe the details of solid-liquid contact area through experiments due to unfavourable optical measurement condition and short impacting time [25]. For instance, it is very difficult to measure the variation of pressure water drops in the impacting process. In recent years, some researchers have resorted Volume of Fluid (VOF) method, a fixed-mesh method, in which the interface between immiscible fluids is modelled as the discontinuity in characteristic function (such as volume fraction) to simulate water droplet impacted on the structured surface [26,27]. The computational studies and parameters considered in these studies are summarised in Table 1. Most of these studies were focused on analysis of the shape evolution, pressure distribution contours and velocity vectors of a droplet as shown in Table 1 . However, the study on pressure variation of air pockets, kinetic and potential energies of a water droplet is still rare.

Furthermore, for a textured surface, static contact angle as a basic index to evaluate the wettability, is highly associated with the surface pattern and intrinsic contact angle of the substrate. Currently, most of studies only simulated a portion of the water droplet impacting process. As shown in Table 1, For studies at high Weber number, the simulated impacting time varied from 8 to $60 \mathrm{~ms}$ [27-29]. At the end of the simulation, the water droplet still in a dynamic process and not reached an equilibrium state. For studies at low Weber number, the water droplet was in micron or submillimetre level, and the impacting time was in $\mu$ s level [27, 28]. Quan investigated the effects of intrinsic contact angle on the dynamic behaviours of droplets and found that substrate with larger intrinsic contact angle has shorten the contact time, and smaller penetrating depth of the liquid [30]. Karthik concluded that analysis of pressure and velocity fields is necessary to exploring the dynamic behaviour of the droplet under different conditions and textured surfaces [31]. However, if the simulation can obtain the static contact 
angle of different substrates, which will help to validate the superhydrophobicity of designed structures.

Zhang et al. investigated the shapes of water drops in the quasi-static and impacting process. The wetting and spreading characteristics of a water drop on a surface were approved to be highly dependent on the height of pillar [32]. The contact angle at equilibrium state increased from $112^{\circ}$ (Wenzel wetting state) to $143^{\circ}$ (Cassie-Baxter state) when pillar heights varied from 18 to $100 \mu \mathrm{m}$ [32]. However, the formation process and mechanism of different wetting state still require further exploration.

Table 1 Summary of the previous work done on impact of drop in a solid surface

\begin{tabular}{|c|c|c|c|c|c|c|c|}
\hline Authors & $\begin{array}{l}\text { Weber } \\
\text { number }\end{array}$ & $\begin{array}{l}\text { Impact } \\
\text { velocity } \\
(\mathrm{m} / \mathrm{s})\end{array}$ & $\begin{array}{l}\text { Contact } \\
\text { angle } \\
\left({ }^{\circ}\right)\end{array}$ & $\begin{array}{l}\text { Water } \\
\text { droplet } \\
\text { diameter } \\
(\mathrm{mm})\end{array}$ & $\begin{array}{l}\text { Computational } \\
\text { time (ms) }\end{array}$ & Substrate & Purposes \\
\hline Li [28] & 93 & 1.505 & 154 & 3 & 13.1 & $\begin{array}{l}\text { Smooth, } \\
\text { Square } \\
\text { Pillars }\end{array}$ & Drop shape \\
\hline $\begin{array}{l}\text { Tembely } \\
{[27]}\end{array}$ & 27.3 & 1.0 & 154 & $2 \mathrm{~mm}$ & 60 & Smooth & $\begin{array}{l}\text { Drop shapes, } \\
\text { freezing process }\end{array}$ \\
\hline Yun [29] & 27 & 1.0 & 155 & 1.97 & 8 & Smooth & $\begin{array}{l}\text { Drop shapes, } \\
\text { momentum }\end{array}$ \\
\hline $\begin{array}{l}\text { Quan } \\
{[30]}\end{array}$ & $0.5-6.2$ & $0.7-3.2$ & 110 & 0.045 & 0.052 & Micro pillars & $\begin{array}{l}\text { Drop shapes, } \\
\text { penetration depth, } \\
\text { pressure } \\
\text { distribution }\end{array}$ \\
\hline $\begin{array}{l}\text { Karthik } \\
{[31]}\end{array}$ & $\begin{array}{l}0.93- \\
6.2\end{array}$ & $1.17-3$ & $\begin{array}{l}114,12 \\
8\end{array}$ & 0.05 & 0.016 & $\begin{array}{l}\text { Square and } \\
\text { circular } \\
\text { pillars }\end{array}$ & $\begin{array}{l}\text { Drop shapes, } \\
\text { pressure } \\
\text { distribution }\end{array}$ \\
\hline $\begin{array}{l}\text { Zhang } \\
\text { [32] }\end{array}$ & $\begin{array}{l}3.4 \mathrm{e}-6, \\
3.4\end{array}$ & $0.001,1$ & 110 & 0.25 & 30 & $\begin{array}{l}\text { Square } \\
\text { Pillars }\end{array}$ & Drop shapes \\
\hline
\end{tabular}

Nanosecond pulsed laser machining process has been proved to be a highly efficient and lowcost approach to machine superhydrophobic structures on stainless steels. In this study, three different substrates with laser ablated Gaussian holes and smooth substrate will be prepared as case studies. The underlying mechanism of different wetting state at both low and high Weber numbers will be extensively examined through VOF simulation. The average pressure of air 
pockets will be investigated to explain the formation process of a superhydrophobic state (i.e. Cassie-Baxter state). Furthermore, the numerical results of static contact angle at equilibrium state will be validated through measured values of surfaces obtained in laser ablation experiment. The dynamic energy transition among kinetic energy and potential energy will be further investigated through numerical simulation. Finally, the high-speed camera test will be carried out to verify the simulation results.

\section{Computational fluid dynamics (CFD) simulation details}

\subsection{Mathematical model of the VOF method}

The VOF method can be applied on two or more immiscible fluids by solving a series of momentum equations and by tracking the volume fraction for every fluid throughout the domain [33]. In the superhydrophobic phenomenon, solid phase (AISI 316L stainless steel), liquid phase (water) and gas phase (air) are three immiscible fluids. Thus, the VOF method can be employed to simulate the impacting behaviour of water droplets on smooth and textured substrates to explore the underlying physics of the superhydrophobic phenomenon.

In this study, the water droplet impacting process is considered to take place at room temperature, and the process is adiabatic. ANSYS-CFD solver was employed in the simulation. The general form of the mass conservation equation is expressed as follows, which is valid for both incompressible and compressible flows.

$$
\frac{\partial \rho}{\partial t}+\nabla \cdot(\rho \vec{v})=S_{m}
$$

where $\rho$ is density, $t$ is time, and $\vec{v}$ is fluid velocity. $S_{m}$ represents the source item, which means the mass added to the continuous phase from another phase or other phase. $S_{m}$ is 0 in this research.

G. K. Batchelor[34] et al. described the conservation of momentum as:

$$
\frac{\partial}{\partial t}(\rho \vec{v})+\nabla \cdot(\rho \vec{v} \vec{v})=-\nabla p+\nabla \cdot(\overline{\bar{\tau}})+\rho \vec{g}+\vec{F}
$$

where $p$ is the static pressure, $\overline{\bar{\tau}}$ is the stress tensor and expressed in Eq. (3). $\vec{g}$ is the acceleration of gravity, $\vec{F}$ is the external body forces.

$$
\overline{\bar{\tau}}=\mu\left[\left(\nabla \vec{v}+\nabla \vec{v}^{T}\right)-\frac{2}{3} \nabla \cdot \vec{v} I\right]
$$


where $\mu$ is the molecular viscosity, $I$ is the unit tensor, and the second term on the right side is the effect of volume dilation.

For a single water droplet, the surface tension has a significant effect on its impacting behaviour. The continuum surface force (CSF) model developed by Brackbill [35] et al. was used in this research to consider the surface tension effect. In the CSF model, the volume force $\left(F_{v o l}\right)$ of surface tension to the VOF numerical is a source term $\vec{F}$ in the Eq. (2). For two phase numerical calculation, the phases are represented by the subscripts 1 and 2 . The volume force $\left(\vec{F}_{v o l}\right)$ can be expressed by Eq. (4). $k_{1}$ is the curvature of first phase and can be expressed by Eq. (5). $\hat{n}_{1}$ is the unit normal as described in Eq. (6).

$$
\begin{gathered}
F_{v o l}=\sigma_{12} \frac{\rho k_{1} \nabla \alpha_{1}}{\frac{1}{2}\left(\rho_{1}+\rho_{2}\right)} \\
k_{1}=\nabla \cdot \hat{n}_{1} \\
\hat{n}_{1}=\frac{\nabla \alpha_{1}}{\left|\nabla \alpha_{1}\right|}
\end{gathered}
$$

where, $\sigma_{12}$ is the surface tension coefficient between two phases, and $\alpha_{1}$ is the volume fraction of the first phase.

The intrinsic contact angle $(\theta)$ provides information about the wettability of an ideal smooth surface. Usually, it comes from the test results. In the VOF simulation, $\theta$ is not imposed on the wall itself, but it is used to adjust the surface normal in cells near the wall [35]. Hence, it results in the adjustment of the curvature of the surface near the wall. The surface unit normal at the live cell next to the wall can be expressed as:

$$
\hat{n}=\hat{n}_{\text {wall }} \cos \theta+\hat{t}_{\text {wall }} \sin \theta
$$

where $\hat{n}_{w a l l}$ and $\hat{t}_{\text {wall }}$ are the unit vectors normal and tangential to the wall, respectively.

The Weber number (We), often used in analysing fluid flows of an interface between two different fluids, is a dimensionless parameter. It represents the ratio of the inertial force to the surface tension force. The Weber number (We) can be expressed as:

$$
W e=\frac{\rho v^{2} l}{\sigma}
$$

where $l$ is its characteristic length, typically the droplet diameter. 


\subsection{Geometry model and computational setup}

In our previous experimental study, the nanosecond laser machining process was developed to improve the hydrophobicity of AISI 316L stainless steel surface [22]. A geometrical model of laser ablated Gaussian microhole was established as shown in Fig. 1 (a). The nanosecond laser beam has a Gaussian intensity profile and hence the profile of laser machined Gaussian hole will also be like a Gaussian curve as shown in Fig.1 (b). In this figure, $p$ is the pitch between two adjacent Gaussian holes, $a$ is the depth of the Gaussian hole, $6 c$ is the width of the Gaussian hole, and $\theta_{\mathrm{CB}}$ is the apparent contact angle of water droplet at Cassie-Baxter state. Gaussian hole can be characterized by Gaussian function as shown in Eq. (9). Thus, the curve between $\pm 3 \mathrm{c}$ was chosen to generate the 3D Gaussian hole by CAD software as shown in Fig.1 (a).

$$
z=-a \times e^{-\frac{x^{2}+y^{2}}{2 c^{2}}}
$$

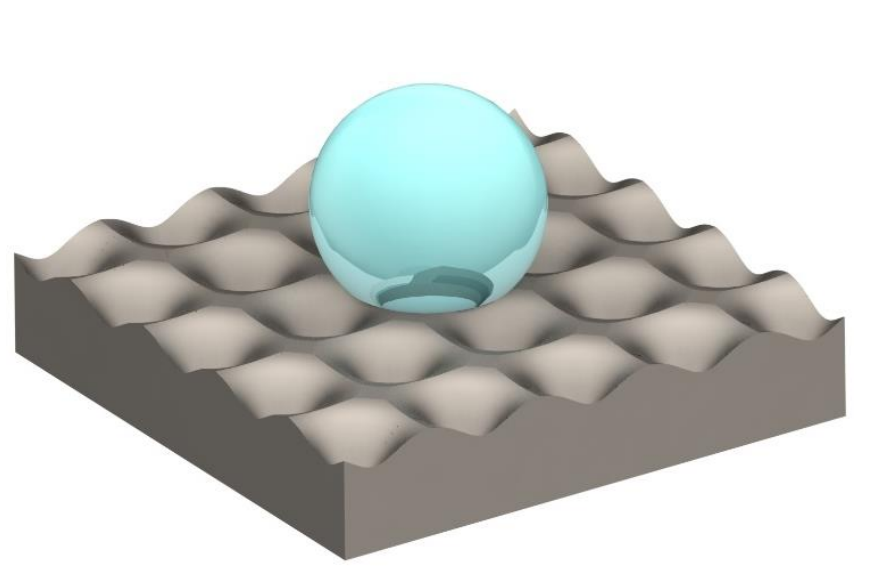

(a)

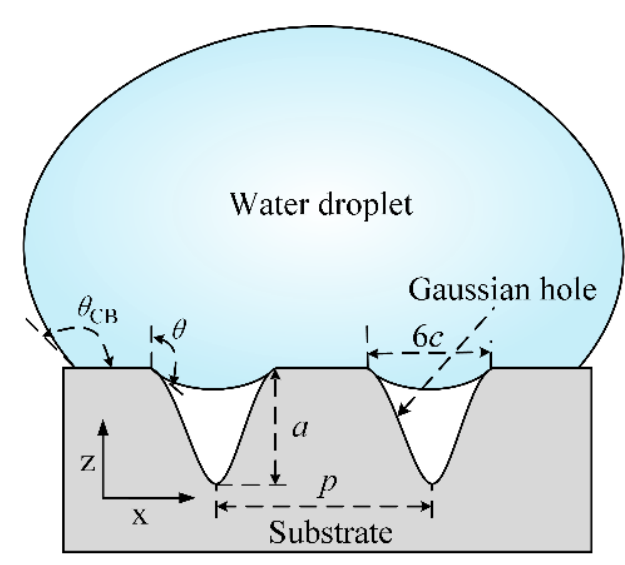

(b)

Fig. 2. Geometrical model of laser machined Gaussian holes at Cassie-Baxter state.

Fig. 1 (a) CAD model of Gaussian hole arrays (b) 2D Mathematical model of laser machined Gaussian holes

The P110 (optimised) and P150 (optimised) have the optimised dimensions same with the previous publication [22], as shown in Table 2. Furthermore, smooth surface and P150 (revised) are employed to simulate the impacting behaviour of water droplets on a smooth surface and structure without the optimised dimensions.

Various efforts have been focused on droplet impact on surfaces with higher impacting velocity. However, it is difficult to predict the final contact angle under higher impacting velocity due to the longer impacting time needed to realise an equilibrium state. In this research, the 
simulation was conducted with low and high Weber numbers. The simulation at low Weber number $(W e=0.026)$ shows the quasi-static process of water droplets, and it can be used to predict the contact angle because the low Weber number takes a short time to reach the equilibrium state. However, the simulation at a high Weber number $(W e=7.26)$ can display the impacting process of water droplets on substrates. The dimensions and initial conditions of the Gaussian hole model for the VOF simulation are shown in Thus, the effect of depth and width on the water droplet impacting behaviour and the final apparent contact angle of microstructures can be investigated from comparison between P150 (revised) and P110 (optimised). And the effect of Gaussian hole's pitch can be investigated from comparison between P150 (revised) and P110 (optimised). As shown in Table 1, the impact velocities of water droplet used in previous studies varied from 0.001 to $3.2 \mathrm{~m} / \mathrm{s}$. Based on the above analysis, the water droplet with a diameter of $2.12 \mathrm{~mm}(5 \mu \mathrm{L})$ has impact velocities of $0.03 \mathrm{~m} / \mathrm{s}$ $(W e=0.026)$ and $0.5 \mathrm{~m} / \mathrm{s}(W e=7.26)$ are used in this study.

Table 2. Specifically, P150 (revised) has a smaller depth $a$ and width $6 c$ than P150 (optimised), and it has a larger pitch if compared with P110 (optimised). Thus, the effect of depth and width on the water droplet impacting behaviour and the final apparent contact angle of microstructures can be investigated from comparison between P150 (revised) and P110 (optimised). And the effect of Gaussian hole's pitch can be investigated from comparison between P150 (revised) and P110 (optimised). As shown in Table 1, the impact velocities of water droplet used in previous studies varied from 0.001 to $3.2 \mathrm{~m} / \mathrm{s}$. Based on the above analysis, the water droplet with a diameter of $2.12 \mathrm{~mm}(5 \mu \mathrm{L})$ has impact velocities of $0.03 \mathrm{~m} / \mathrm{s}$ $(W e=0.026)$ and $0.5 \mathrm{~m} / \mathrm{s}(W e=7.26)$ are used in this study.

Table 2 Dimensions and initial conditions of the Gaussian hole model for VOF simulation.

\begin{tabular}{|c|c|c|c|c|c|c|c|}
\hline $\begin{array}{l}\text { Case } \\
\text { run }\end{array}$ & Name & Surface pattern & $P(\mu \mathrm{m})$ & $a(\mu \mathrm{m})$ & $6 c(\mu \mathrm{m})$ & $\begin{array}{l}\text { Impacting } \\
\text { velocity } \\
(\mathrm{m} / \mathrm{s})\end{array}$ & We \\
\hline 1 & Smooth & Smooth & 0 & 0 & 0 & 0.03 & $\begin{array}{l}0.02 \\
6\end{array}$ \\
\hline 2 & P150 (revised) & $\begin{array}{l}\text { Gaussian hole } \\
\text { arrays }\end{array}$ & 150 & 86 & 114.6 & 0.03 & $\begin{array}{l}0.02 \\
6\end{array}$ \\
\hline 3 & P150 (optimised) & $\begin{array}{l}\text { Gaussian hole } \\
\text { arrays }\end{array}$ & 150 & 110.9 & 153.6 & 0.03 & $\begin{array}{l}0.02 \\
6\end{array}$ \\
\hline 4 & P110 (optimised) & $\begin{array}{l}\text { Gaussian hole } \\
\text { arrays }\end{array}$ & 110 & 86 & 114.6 & 0.03 & $\begin{array}{l}0.02 \\
6\end{array}$ \\
\hline
\end{tabular}




\begin{tabular}{|c|c|c|c|c|c|c|c|}
\hline 5 & P150 (revised) & $\begin{array}{l}\text { Gaussian hole } \\
\text { arrays }\end{array}$ & 150 & 86 & 114.6 & 0.5 & 7.26 \\
\hline 6 & P150 (optimised) & $\begin{array}{l}\text { Gaussian hole } \\
\text { arrays }\end{array}$ & 150 & 110.9 & 153.6 & 0.5 & 7.26 \\
\hline 7 & P110 (optimised) & $\begin{array}{l}\text { Gaussian hole } \\
\text { arrays }\end{array}$ & 110 & 86 & 114.6 & 0.5 & 7.26 \\
\hline
\end{tabular}

The computational setup in the VOF simulation is shown in Table 3. The solid substrate material is steel. The wall adhesion angle is same as the initial contact angle on the smooth surface.

Table 3 Computational Setup in VOF simulation

\begin{tabular}{ll}
\hline Parameter & Setting/Value \\
\hline Primary Phase & Air \\
Second Phase & Water \\
Solid & Steel \\
Calculation type & Transient model \\
Calculation model & Volume of fluid \\
Diameter of water droplet $\left(D_{0}\right)$ & $2.12 \mathrm{~mm}(5 \mu \mathrm{L})$ \\
Surface tension & $0.073 \mathrm{~N} / \mathrm{m}$ \\
Wall adhesion angle & $105^{\circ}$ \\
Time step & $2.5^{*} 10^{-5} \mathrm{~s}$ \\
\hline
\end{tabular}

Since the Gaussian hole model and the water droplet are symmetric, a one-quarter symmetric model was used in this research. The 3D computational domain and boundary conditions of the Gaussian hole model are illustrated in Fig. 2 (a). The dimension of the computational domain is $4 \times 4 \times 4 \mathrm{~mm}^{3}$. All the side walls are set as a symmetry boundary condition. A rigid no-slip wall boundary condition with a static contact angle $105^{\circ}$ is imposed at the structured surface. The initial state of the water droplet is shown in Fig. 2 (b). 

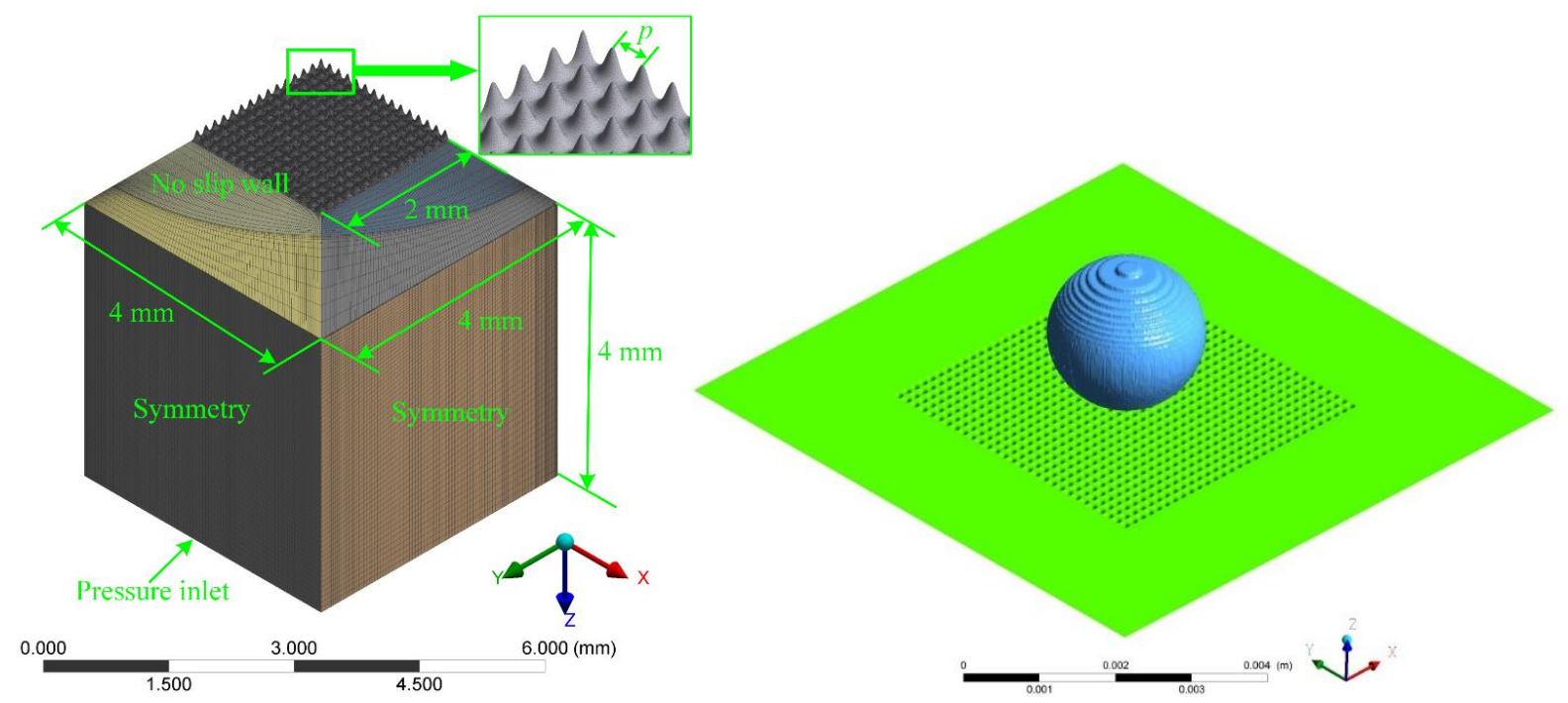

Fig. 2 (a) 3D geometry model of fluid domain based on Gaussian characters (b) Initial state of water droplet

\section{CFD simulation: results and discussions}

\subsection{Dynamic behaviour of the water droplet on varied substrates at a Weber number of} 0.026

In this section, low impacting velocity of $0.03 \mathrm{~m} / \mathrm{s}$ is employed as the initial condition to impact the different substrates to predict the static contact angle at the equilibrium state.

In general, when the water droplet impacts a solid surface, two stages spreading and retracting are cyclically performed until realising an equilibrium state. As shown in Fig. 3 (a) and (b), the droplet expanded rapidly in the radial direction from $0.05 \mathrm{~ms}$ to $10 \mathrm{~ms}$, and the water droplet height decreased continuously until the vertical velocity component was reduced to 0 , which is called the spreading stage. The retracting stage started at $10 \mathrm{~ms}$ and lasted until $15 \mathrm{~ms}$, and the droplet moved upward due to the surface tension effect. Fig. 3 (b) shows the variations in pressure and velocity vector. During the spreading stage, the water droplet's velocity vectors moved downward and to the outside. Inversely, the velocity vectors at the retracting stage moved upward and to the inside. After multiple oscillations, the water droplet reached an equilibrium state at $200 \mathrm{~ms}$. Fig. 3 (c) shows that the water droplet's pressure distribution at $200 \mathrm{~ms}$ complied with the law of pressure variation in a static fluid, which states that pressure increases with depth. Furthermore, the velocity vector was lower than $0.05 \mathrm{~m} / \mathrm{s}$ at $200 \mathrm{~ms}$, which is negligible and can be regarded as an equilibrium state. 


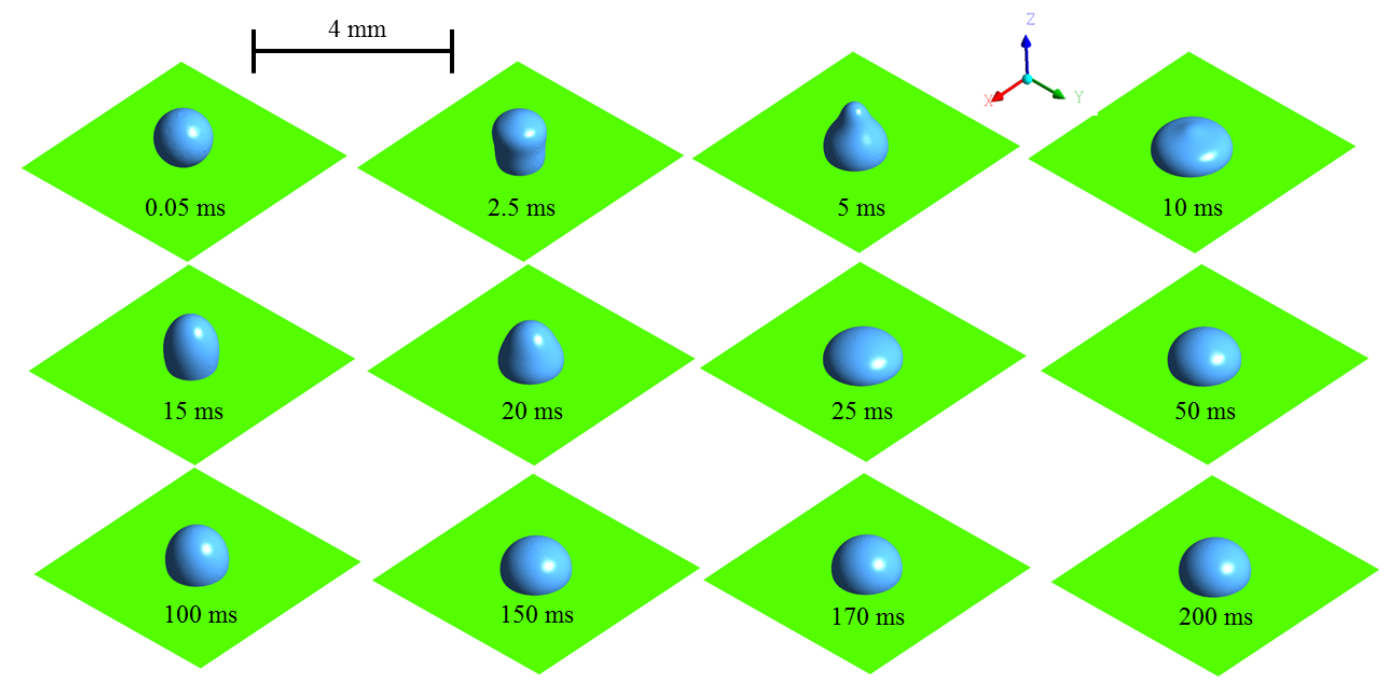

(a)

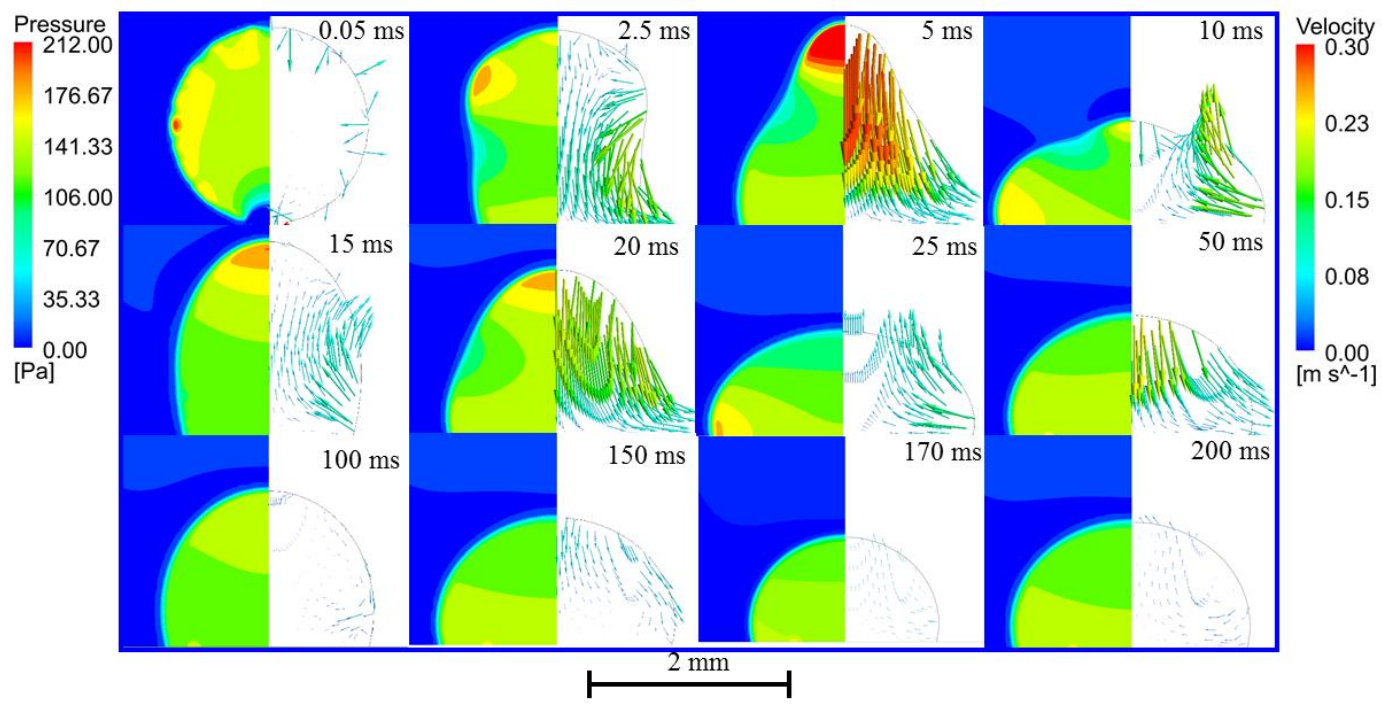

(b)

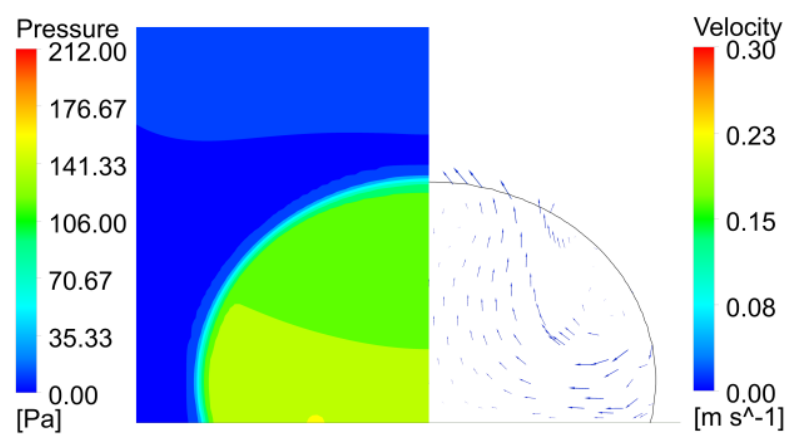

(c) 
Fig. 3 A series of images of a water droplet impact on the specimen with a smooth surface at a low impact velocity of $0.03 \mathrm{~m} / \mathrm{s}$ (We $=0.026$, Case 1 ): (a) shape of the water droplet, (b) static pressure and velocity vector and (c) static pressure and velocity vector at $200 \mathrm{~ms}$.

The dynamic droplet shape and its cloud map of pressure-velocity vector for the P150 (revised) specimen at We 0.026 are shown in Fig. 4. When the water droplet started to contact the smooth surface, there was only one rebound process, between $0 \mathrm{~ms}$ and $5 \mathrm{~ms}$, as shown in Fig. 4 (a) and (b). Then, the water droplet's height decreased gradually. More details of the CassieWenzel transition process are shown in Fig. 4 (c). When the water droplet started to contact the substrate, the water droplet was in a metastable Cassie-Baxter state, between $0 \mathrm{~ms}$ to $10 \mathrm{~ms}$. The static pressure shows an increasing trend due to the air pocket formed under the water droplet. After $10 \mathrm{~ms}$, the velocity vector is distributed at the bottom of the water droplet (Fig. 4 (b)), which means the transition from a Cassie-Baxter state to a Wenzel state. The intermediate state at $50 \mathrm{~ms}$ proves this inference (Fig. 4 (c)). From $50 \mathrm{~ms}$ to $200 \mathrm{~ms}$, with the decrease of the velocity vector, the water penetrated the Gaussian hole and reached an equilibrium state. The water almost entirely penetrated the structures, and the wetting transition finished at $200 \mathrm{~ms}$, even though a negligible amount of air was still trapped at the bottom of the structures. Therefore, the water droplet was impaled on the microstructures and showed a Wenzel state at $200 \mathrm{~ms}$.

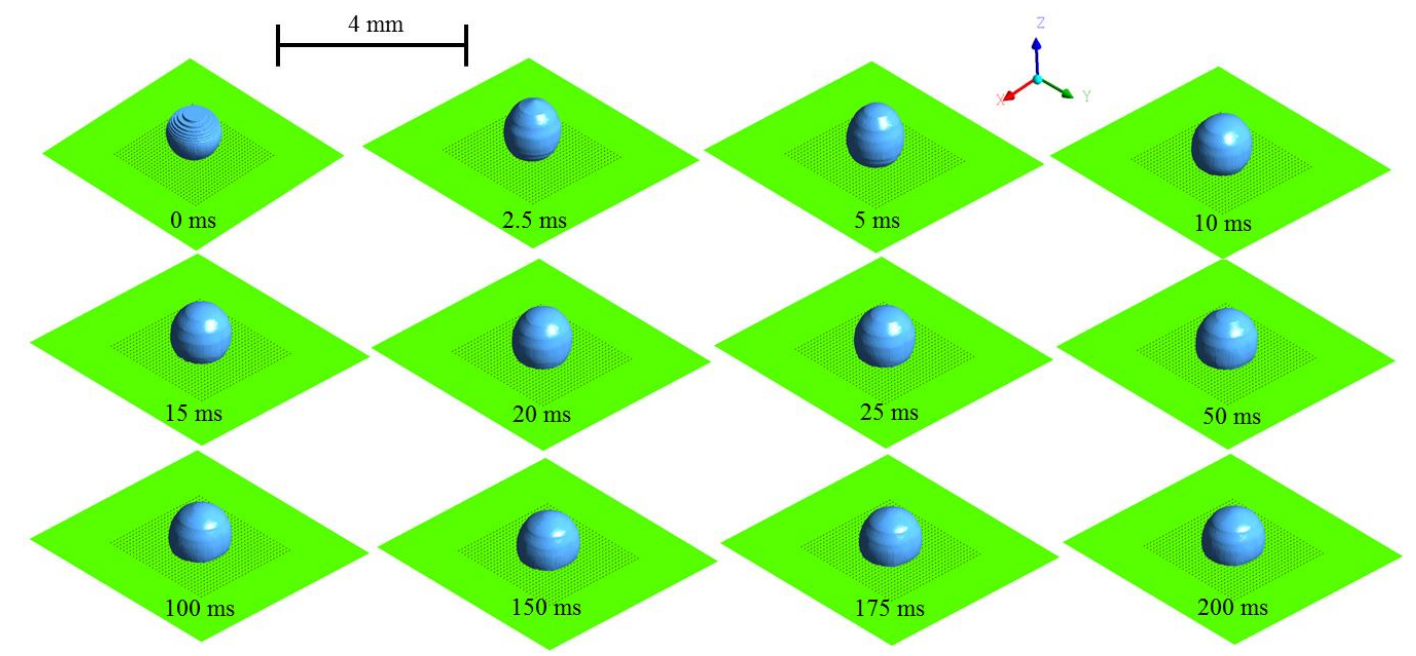

(a) 


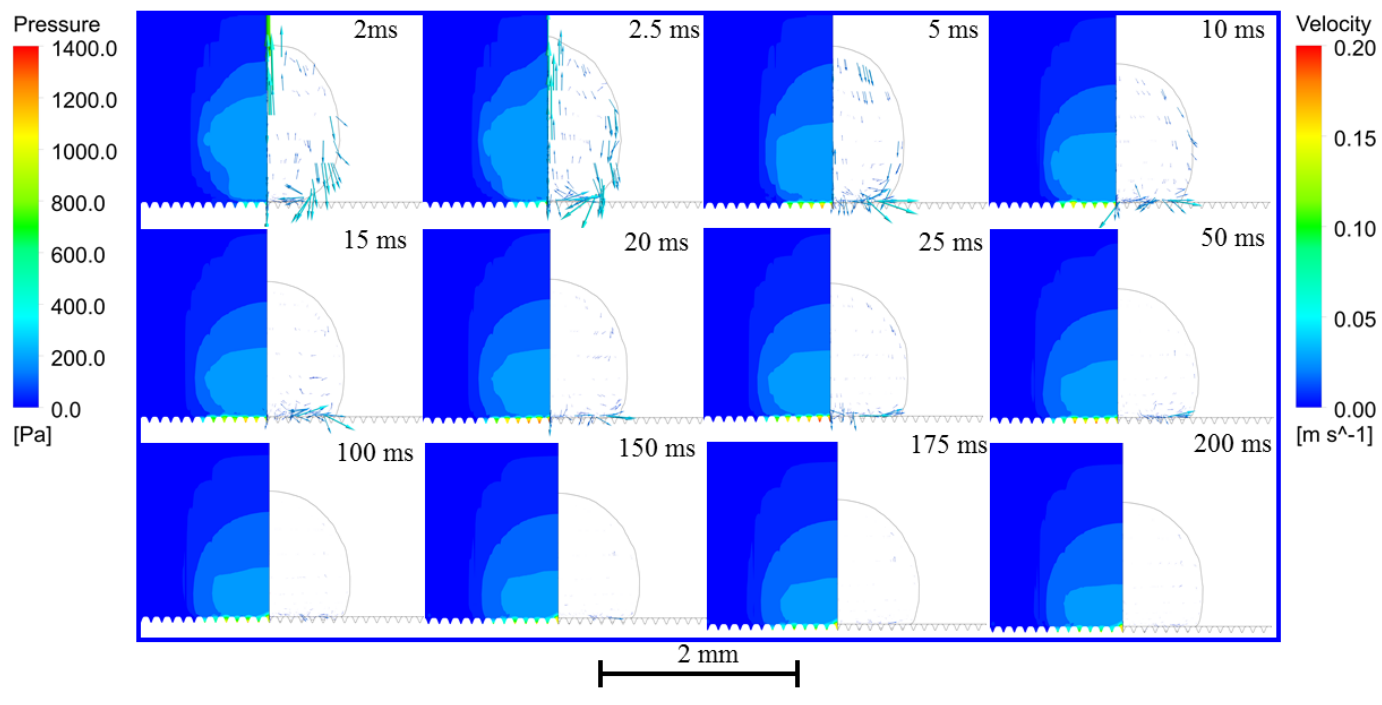

(b)
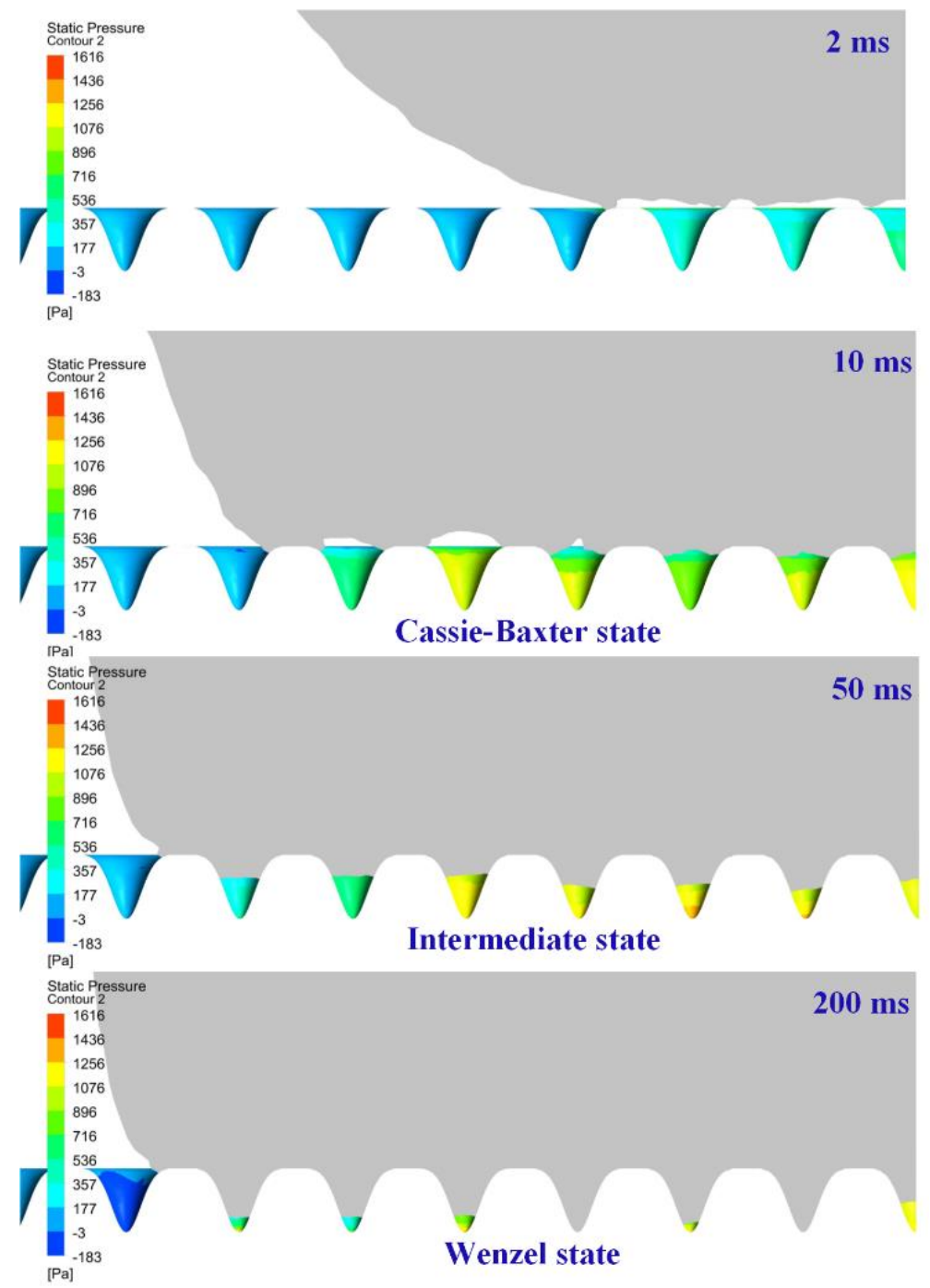

(c) 
Fig. 4 A series of images of a water droplet impact on a specimen with Gaussian hole arrays P150 (revised) at a low impact velocity of $0.03 \mathrm{~m} / \mathrm{s}(W e=0.026$, Case 2$)$ : (a) shape of water droplet, (b) static pressure and velocity vector and (c) Cassie-Wenzel transition process and pressure distribution of Gaussian holes.

The dynamic droplet shapes and its cloud map of pressure-velocity vector for the P150 (optimised) specimen at We 0.026 are shown in Fig. 5. The P150 (optimised) substrate has optimised dimensions, that produce superhydrophobicity with a predicted contact angle of $152.6^{\circ}$, according to the designed results. As shown in Fig. 5 (a) and (b), the water droplet had both horizontal and vertical shape deformation in the spreading process. Furthermore, after 10 $\mathrm{ms}$, the radial velocity vectors were more notable when compared with the vertical direction. The water droplet shape significantly changed in a radial direction due to the water being unable to penetrate the microstructures.

Fig. 5 (c) shows more details of the water droplet state and air pocket pressure. A large volume of air was trapped at the bottom of the structures, resulting in lower adhesion force and making the water droplet stay in a Cassis-Baxter state before $100 \mathrm{~ms}$. However, the water droplet penetrates the Gaussian holes until realising the balance among weight, Laplace pressure and air pocket pressure between $100 \mathrm{~ms}$ and $200 \mathrm{~ms}$. Furthermore, the water droplet shows an intermediate state of Cassie-Baxter and Wenzel state on P150 (optimised) at $200 \mathrm{~ms}$.

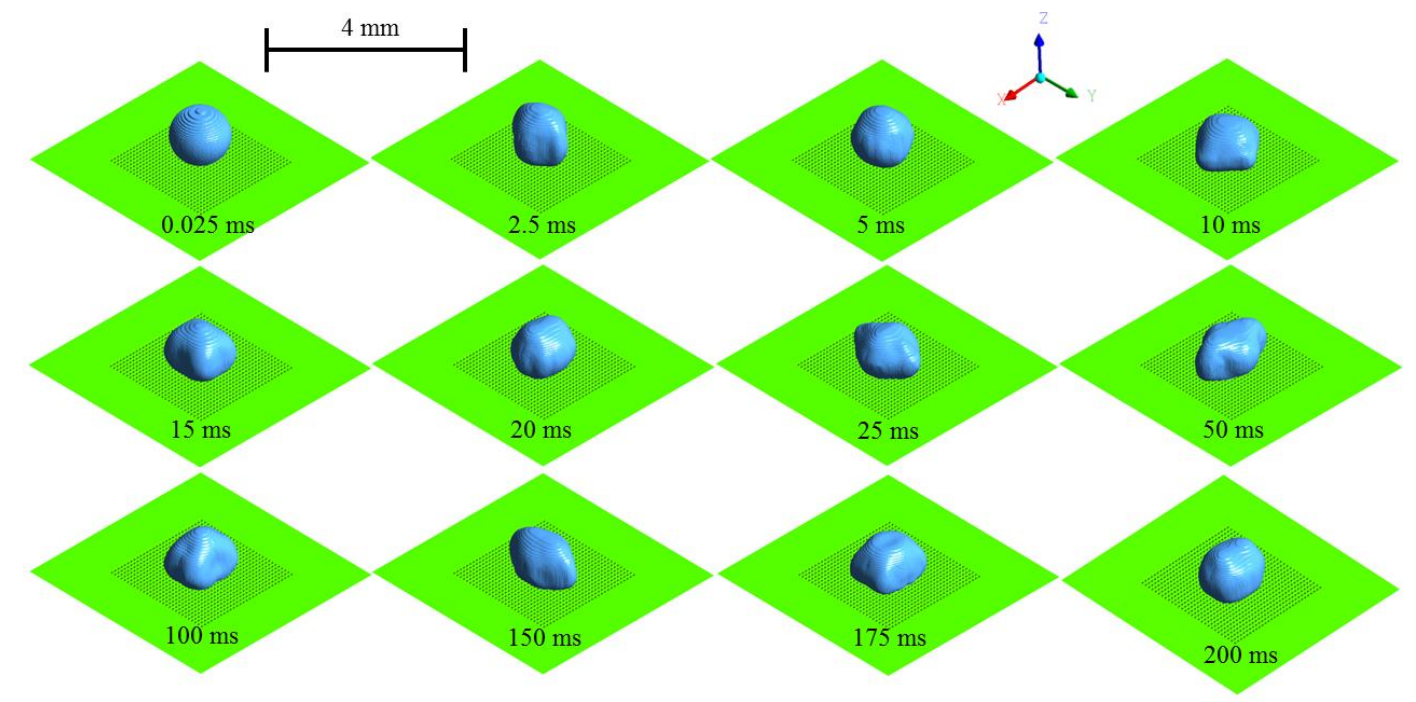

(a) 


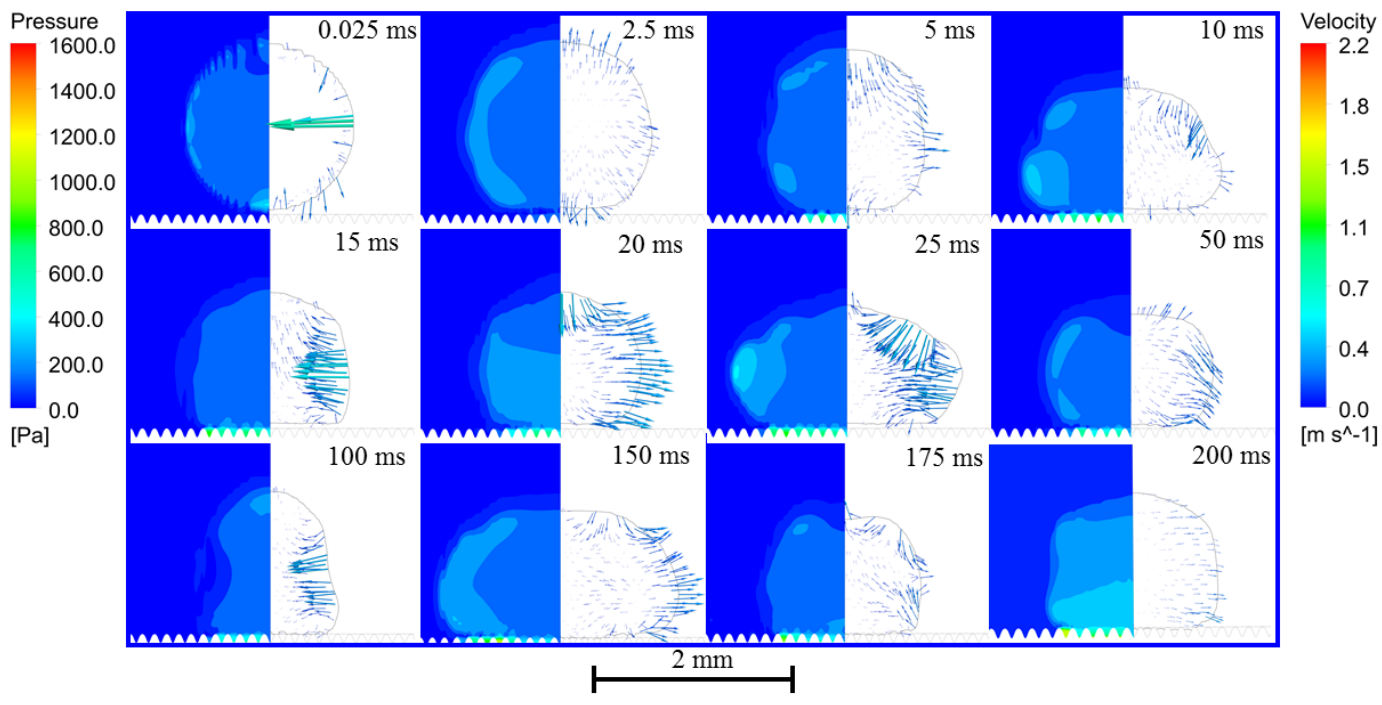

(b)

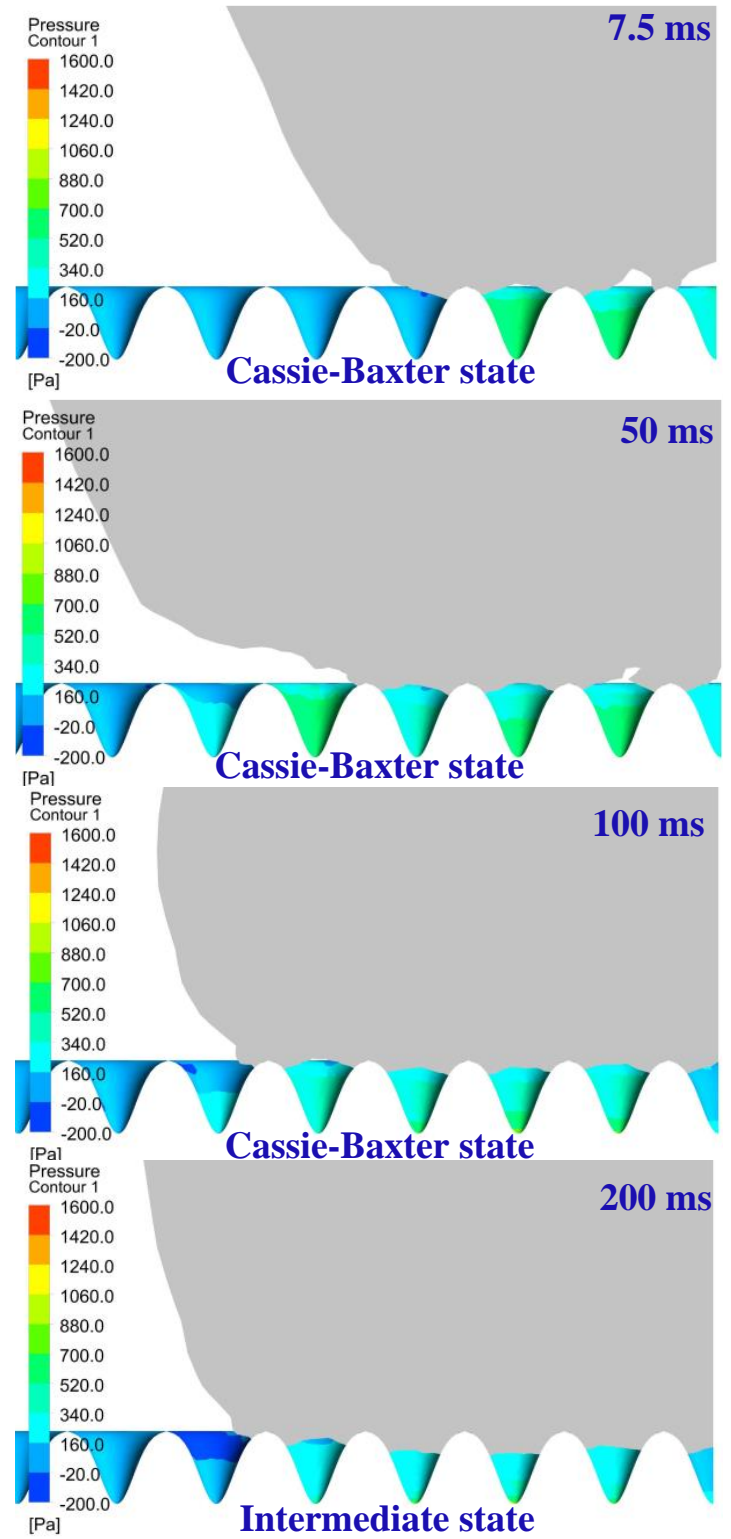

(c) 
Fig. 5 A series of images of a water droplet impact on the specimen with Gaussian hole arrays P150 (optimised) at a low impact velocity of $0.03 \mathrm{~m} / \mathrm{s}(W e=0.026$, Case 3$)$ : (a) shape of water droplet, (b) static pressure and velocity vector and (c) Cassie-Wenzel transition process and pressure distribution of Gaussian holes.

Fig. 6 shows the dynamic droplet shape and its cloud map of pressure-velocity vector for the P110 (optimised) specimen at a Weber number of 0.026. The P110 (optimised) substrate has optimised dimensions, that produce superhydrophobicity with a predicted contact angle of $153.8^{\circ}$, according to the designed results. Similar to P150 (optimised), the water droplet had both horizontal and vertical shape deformations in the spreading process. Furthermore, after 10 $\mathrm{ms}$, the radial velocity vectors were more notable when compared with the vertical direction. Thus, the water droplet shape significantly changed in a radial direction due to the water droplet being unable to wet the Gaussian holes. More details of the water droplet state and air pocket pressure are shown in Fig. 6 (c). A large volume of air was trapped at the bottom of the structures, which resulted in the water droplet having a Cassis-Baxter state on the substrate.

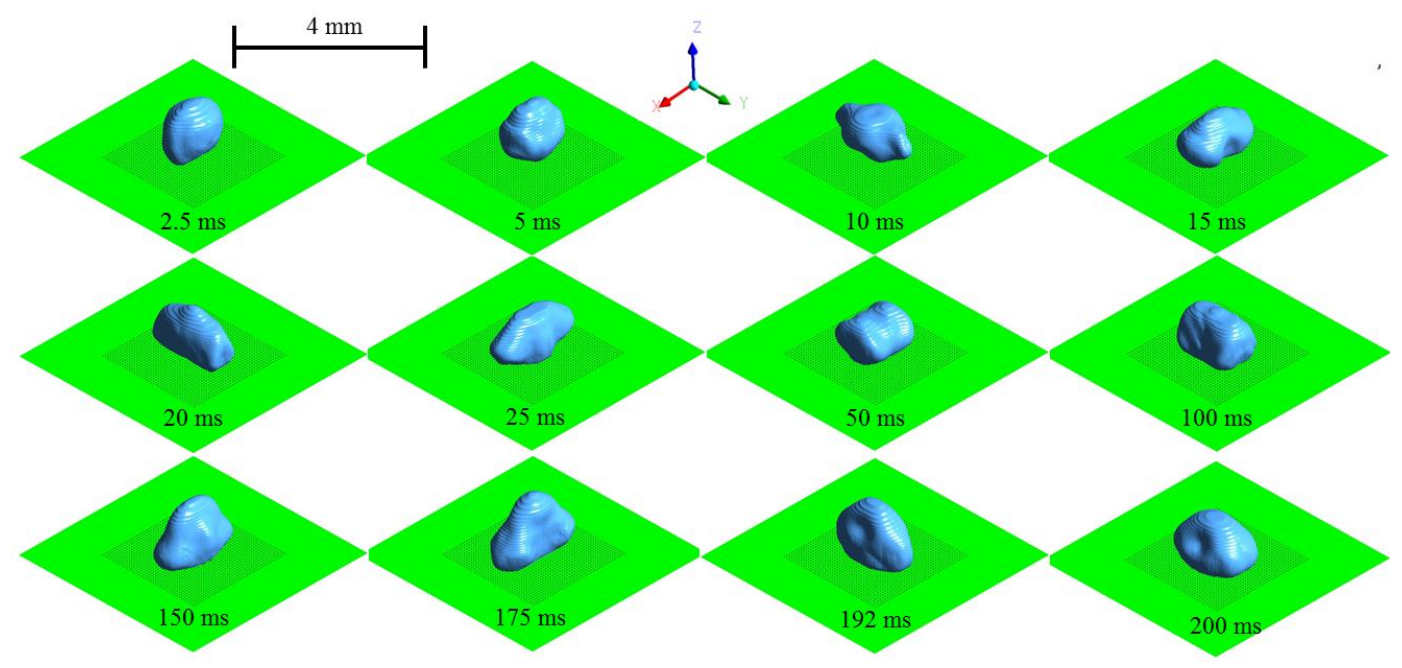

(a) 


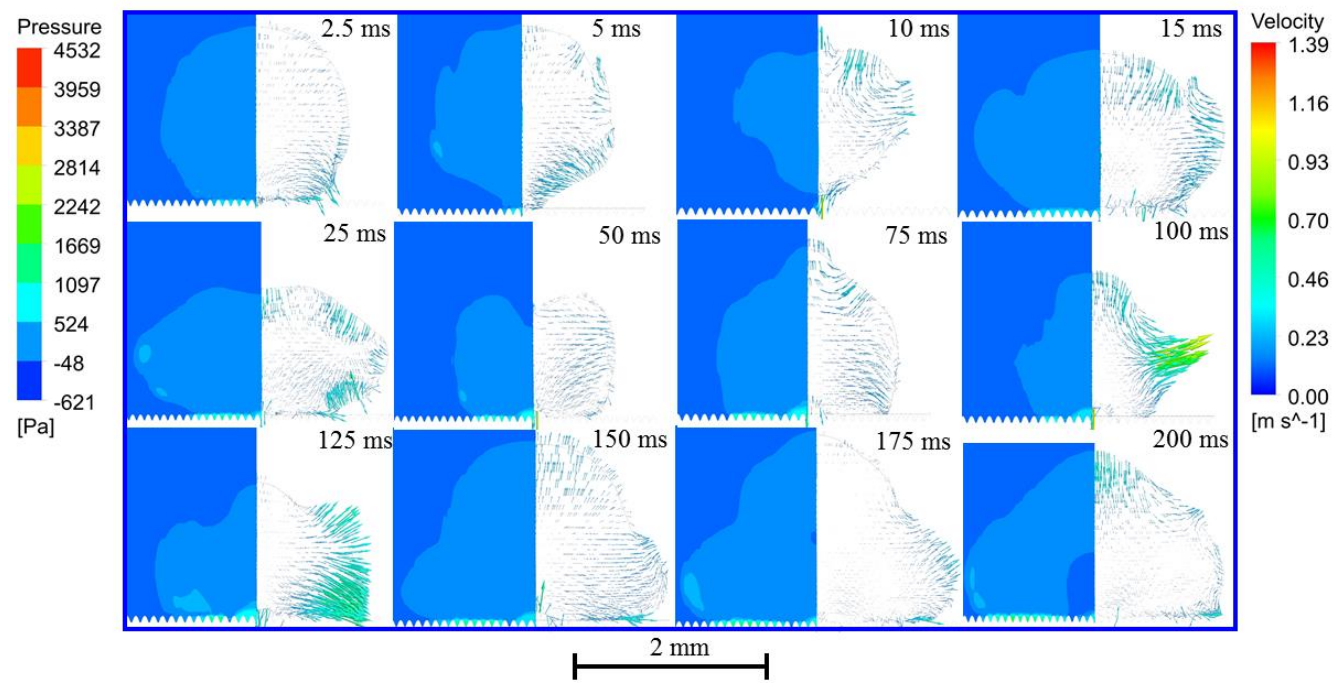

(b)

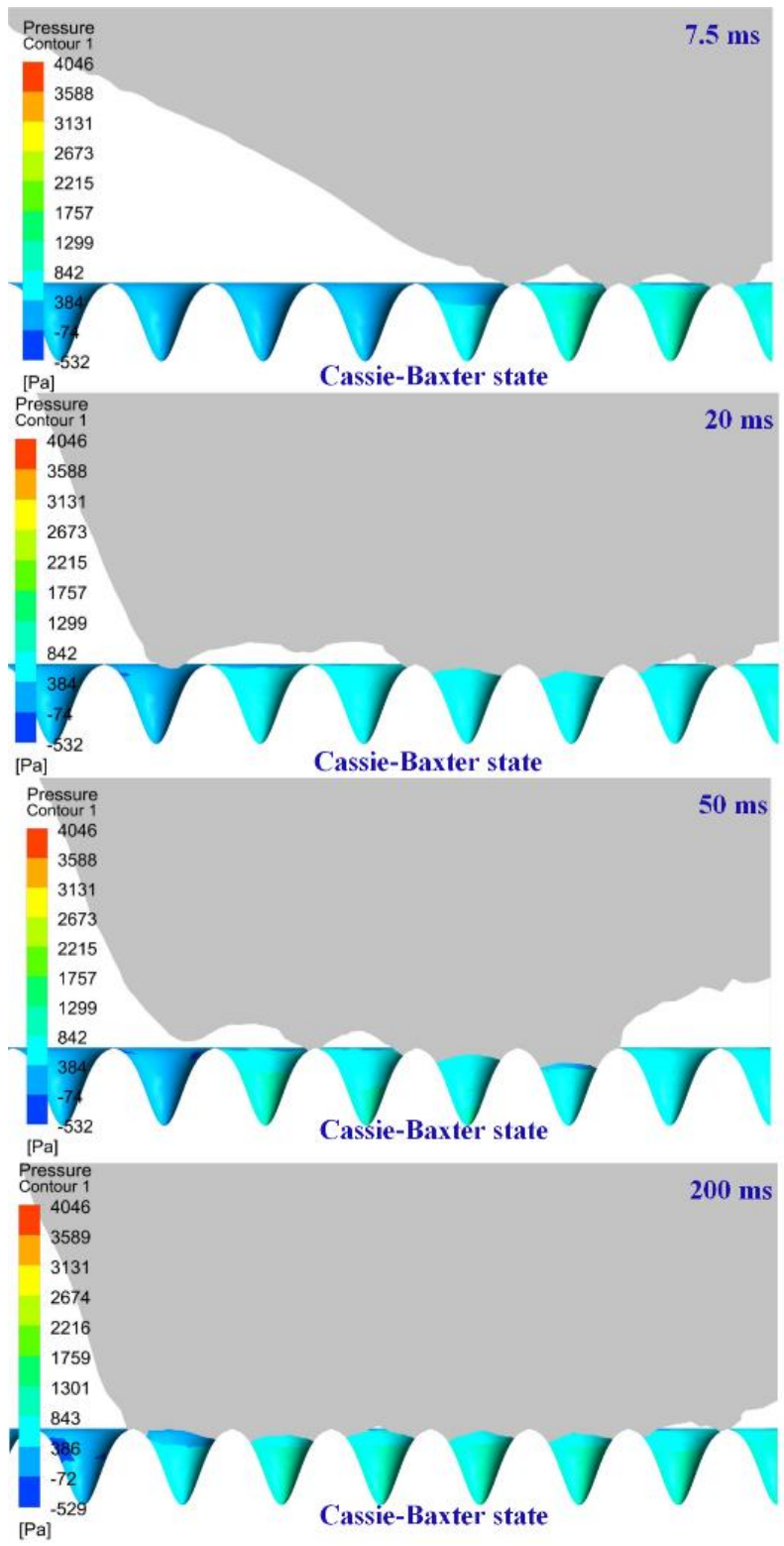

(c) 
Fig. 6 A series of images of a water droplet impact on the specimen with Gaussian hole arrays P110 (optimised) at a low impact velocity of $0.03 \mathrm{~m} / \mathrm{s}(W e=0.026$, Case 4$)$ : (a) shape of water droplet, (b) static pressure and velocity vector and (c) Cassie-Wenzel transition process and pressure distribution of Gaussian holes.

In the impacting process of the water droplets on the substrates, the droplet's shape varied with time in both horizontal and vertical directions. Thus, two dimensionless coefficients (i.e. dimensionless diameter $D^{*}$ and dimensionless height $h^{*}$ ) were defined to quantitatively evaluate the impacting process of a water droplet on the substrates as follows:

$$
\begin{aligned}
D^{*} & =\frac{D}{D_{0}} \\
h^{*} & =\frac{h}{D_{0}}
\end{aligned}
$$

where $D_{0}$ is the initial diameter of the water droplet $(2.12 \mathrm{~mm}$ for a $5 \mu \mathrm{L}$ water droplet), $D$ is the instantaneous maximum diameter of the water droplet, and $h$ is the instantaneous maximum height of water droplet, which is the distance from the top of the water droplet to the solid substrate.

Fig. 7 (a) presents the dimensionless height $h^{*}$ of the droplet for four cases at We 0.026 to explore the hydrophobic and superhydrophobic phenomenon. At a low Weber number, $h^{*}$ can reflect the water droplet's wetting state and dynamic behaviour. For a smooth surface, the droplet's oscillation exhibited a form of damping vibration, and a decreasing trend of oscillation amplitude was observed. For specimen P150 (revised), there was no distinct vibration, and $h^{*}$ was decreased gradually with the wetting process. However, for superhydrophobic substrates P150 (optimised) and P110 (optimised), $h *$ was larger than the other two cases because the water could not wet the structures. Knowledge of the average pressure of the air pockets underneath the droplet during its interaction helps reveal the root mechanism behind different wetting states (Fig. 7 (b)). For both substrates, the average air pockets' pressure shows an increasing trend in the initial formation stage due to air being gradually trapped in the structures. However, the air pockets for P150 (revised) are metastable, and the average pressure of the air pockets remains constant for an extremely short time and then starts decreasing with the wetting transition process from the Cassie-Baxter state to the Wenzel state. For P150 (optimised) and P110 (optimised), the air pockets' pressure increases at first and then remains stable, which helps prevent the water from penetrating the micro Gaussian holes. However, the stable pressure of P150 (optimised) is smaller than that of P110 
(optimised); thus this demonstrates an intermediate state and not a Cassie-Baxter state, like the one found with P110 (optimised).

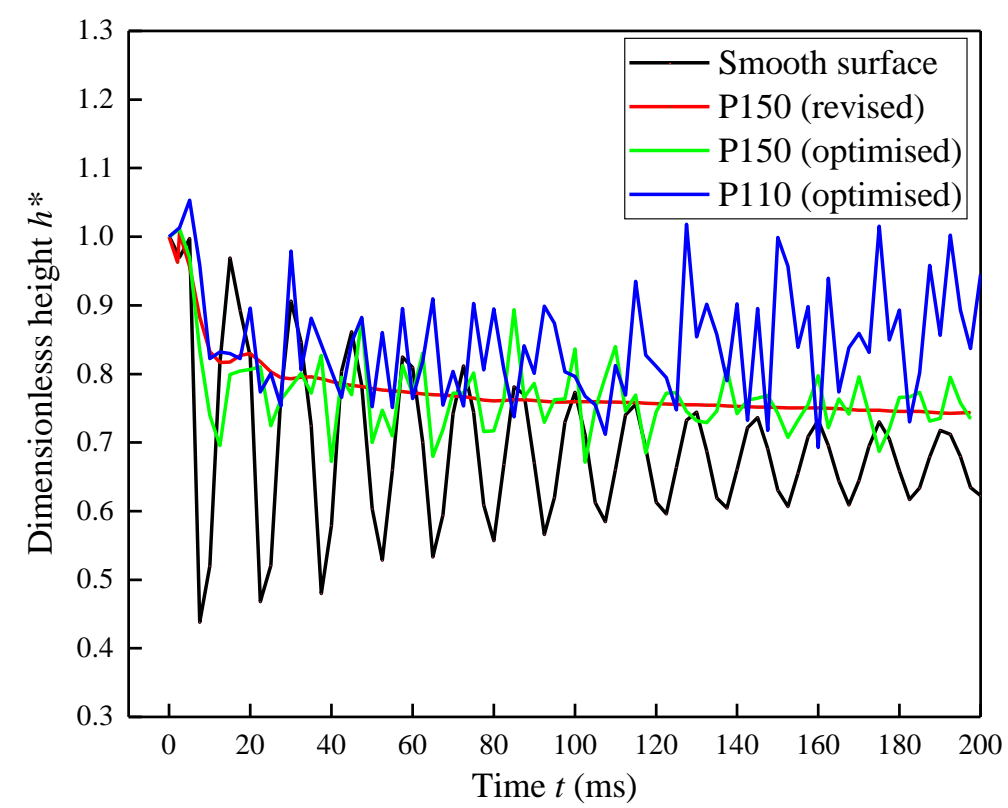

(a)

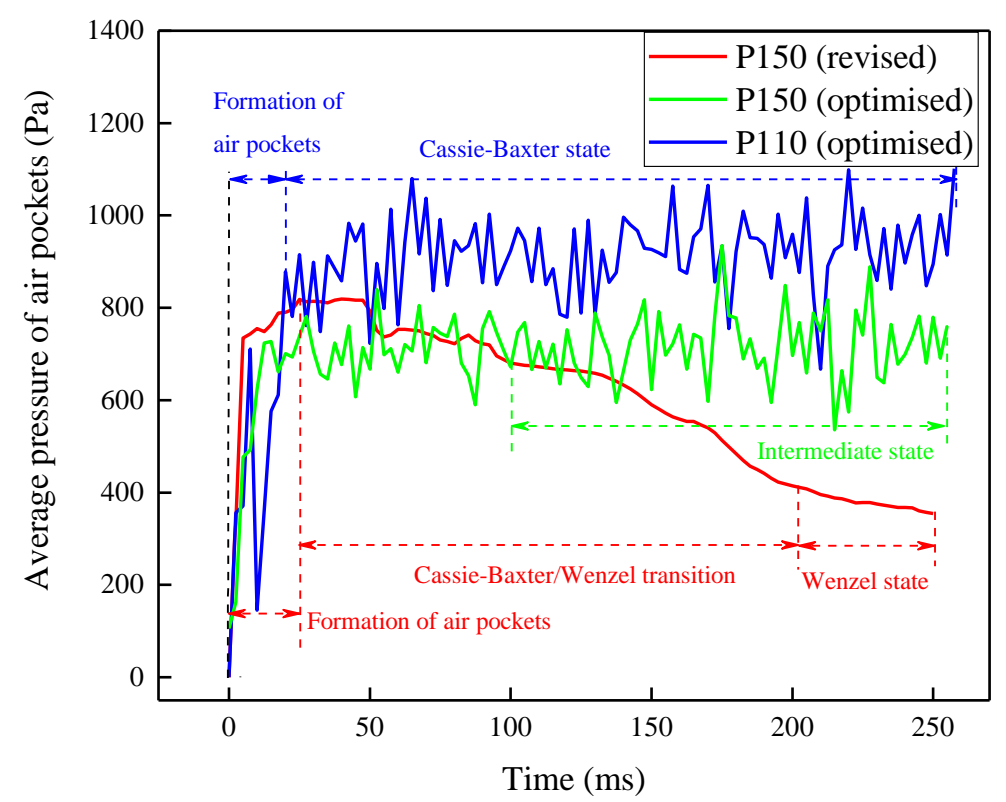

(b)

Fig. 7 (a) Time evolution of $h^{*}$ of water drops $(W e=0.026)$; (b) variations of average air pockets' pressure $(W e=0.026)$.

Furthermore, the variations in the water droplet's mechanical energy are highly related to the practical applications of a superhydrophobic surface. For example, the self-cleaning property is highly associated with the water droplet's kinetic energy $\left(E_{\mathrm{k}}\right)$, and gravitational potential energy $\left(E_{\mathrm{g}}\right)$ can reflect the wetting state. Simulation results are used to calculate the kinetic 
energy and gravitational potential energy. The water droplet's gravitational potential energy $\left(E_{\mathrm{g}}\right)$ and kinetic energy $\left(E_{\mathrm{k}}\right)$ can be calculated by summing all the related elements, which can be expressed as:

$$
\begin{gathered}
E_{\mathrm{g}}=\sum_{1}^{n}\left(\rho_{\mathrm{w}} V_{\text {element }} \alpha_{2}\right) g h_{\text {element }} \\
E_{\mathrm{k}}=\sum_{1}^{n} \frac{1}{2}\left(\rho_{\mathrm{w}} V_{\text {element }} \alpha_{2}\right) \vec{v}^{2}
\end{gathered}
$$

where $n$ is the total number of the element that contains the water phase, $\rho_{\mathrm{w}}$ is the density of water, $V_{\text {element }}$ is the volume of the element, $\alpha_{2}$ is the volume fraction of the water phase, $g$ is the gravitational acceleration equal to $9.81 \mathrm{~m} / \mathrm{s}^{2}, h_{\text {element }}$ is the height of every element and $\vec{v}$ is the water velocity in every element.

The simulation results of potential energy and kinetic energy at We 0.026 are shown in Fig. 8 . At the initial time, the water droplet had maximum gravitational potential energy in all cases (Fig. 8 (a)) due to the water droplet being in the highest position and having no contact with the substrate. With increased time, the gravitational potential energy starts transferring to kinetic energy; thus, the kinetic energy had a significant increase at the initial moment accompanied with the falling and spreading of the water droplet (Fig. 8 (b)). The potential and kinetic energies showed declining trends and no oscillation in the gradual wetting process in the hydrophobic substrate P150 (revised). The water droplet penetrated the microstructure; hence, the initial kinetic energy and potential energy transferred to surface energy due to the increased solid-liquid contact area of the water droplet's Wenzel state on P150 (revised). For P150 (optimised), the water droplet cannot fully penetrate the microstructure and shows an intermediate wetting state, and the initial kinetic energy and potential energy of the water droplet is difficult to transfer to surface energy, which results in the water droplet having higher kinetic energy and potential energy. The superhydrophobic substrate (P110 (optimised)) had the maximum potential energy and kinetic energy in the whole impacting process due to the water droplet having a Cassie-Baxter state on the substrate, which has the smallest solid-liquid contact area. 


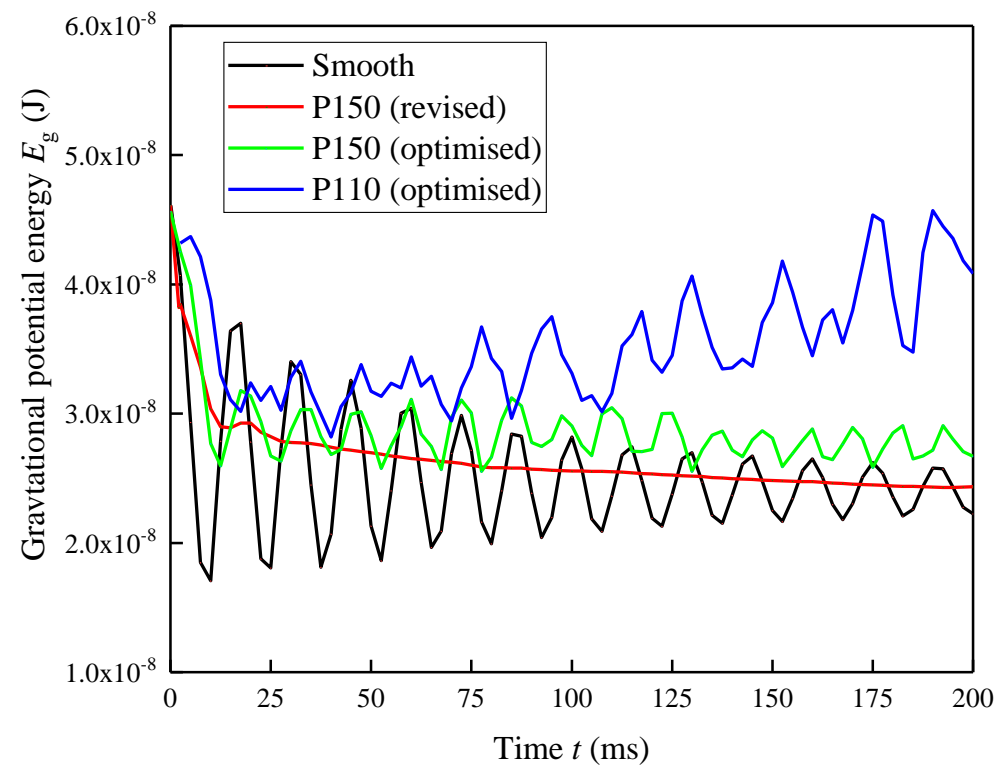

(a)



(b)

Fig. 8 The variations in the water droplet's (a) gravitational potential energy $\left(E_{\mathrm{g}}\right)$ and (b) kinetic energy $\left(E_{\mathrm{k}}\right)$.

Fig. 9 shows the simulated contact angles of a water droplet at $200 \mathrm{~ms}$, based on image analysis software (Dimizer), to evaluate the hydrophobic property of four different substrates. The average contact angles were $105.6^{\circ}$ for the smooth surface and $130.95^{\circ}$ for the $\mathrm{P} 150$ (revised) substrate. The measured apparent contact angle for the P150 (optimised) and the P110 (optimised) substrates were $151.1^{\circ}$ and $157.6^{\circ}\left(>150^{\circ}\right)$ respectively, which classifies them as 
superhydrophobic surfaces. Furthermore, stable air pockets can be observed under the bottom of a water droplet in both.

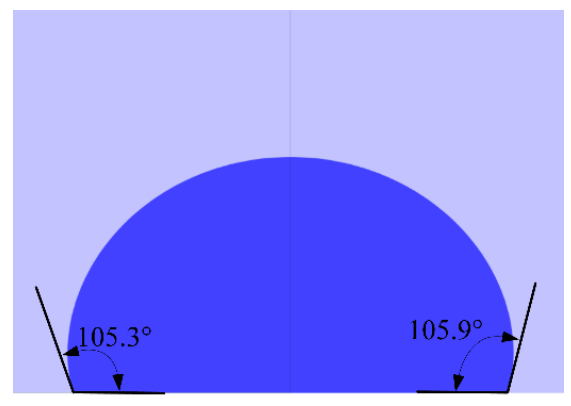

(a)

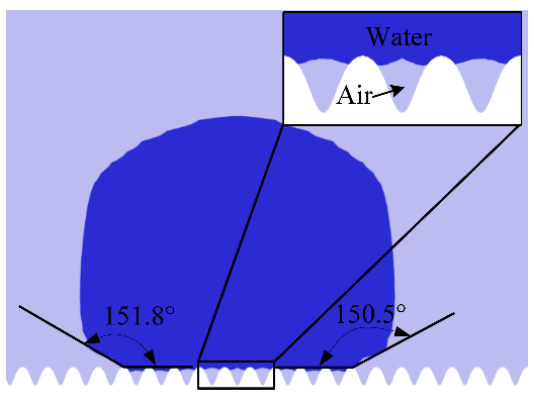

(c)

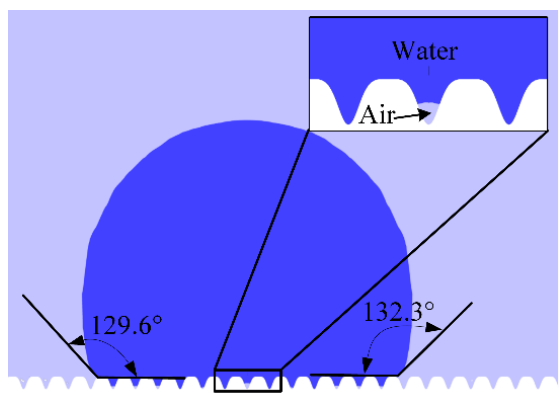

(b)

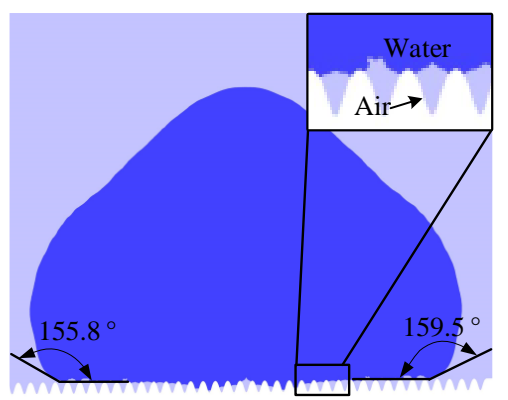

(d)

Fig. 9 Simulated contact angle of water droplet at $200 \mathrm{~ms}$ for (a) smooth surface, (b) P150 (revised), (c) P150 (optimised) and (d) P110 (optimised).

\subsection{Impacting behaviour of a water droplet on varied substrates at Weber number of 7.26}

Section 3.1 explored the underlying mechanism of a water droplet during a quasi-static impacting process. In this section, a medium impacting velocity of $0.5 \mathrm{~m} / \mathrm{s}$ is applied to explore the dynamic rebounding behaviour of water droplets on different substrates. After the droplets impact the substrates, four stages of spreading, recoiling, rebounding and falling are performed until the energy is dissipated. Fig. 10 displays the 3D shapes and velocity vectors of a water droplet on various substrates at different times. In the spreading stage, the water droplet moves downward, which results in water flowing outward along the radial direction. At $2 \mathrm{~ms}$, the water droplet has a maximum velocity of $0.89 \mathrm{~m} / \mathrm{s}$ on substrate P150 (revised), and of $0.64 \mathrm{~m} / \mathrm{s}$ and 0.72 m/s on P150 (optimised) and P110 (optimised), respectively. As shown in Fig. 10 (a), with further increased time, the water droplet has an increased diameter; however, the transverse velocity at the edge of the droplet gradually decreases. Then, the centre of the water droplet keeps going down, as shown at $3 \mathrm{~ms}$, due to the impact of inertia force. However, the water droplet's edge has an upward velocity due to the extrusion force from the water at the 
centre and lower position. As shown in the shape of the water droplet at $6 \mathrm{~ms}$, the water at the central axis has a vertical upward velocity at the recoiling stage. As shown in Fig. 10 (b) and (c), the water droplet shows similar behaviour on P150 (optimised) and P110 (optimised) substrates. However, for substrate P150 (revised), the water detaches from the substrate at 16 ms, while for P150 (optimised) and P110 (optimised), the water droplet requires just $13.4 \mathrm{~ms}$ and $12 \mathrm{~ms}$, respectively, to detach from the surface.

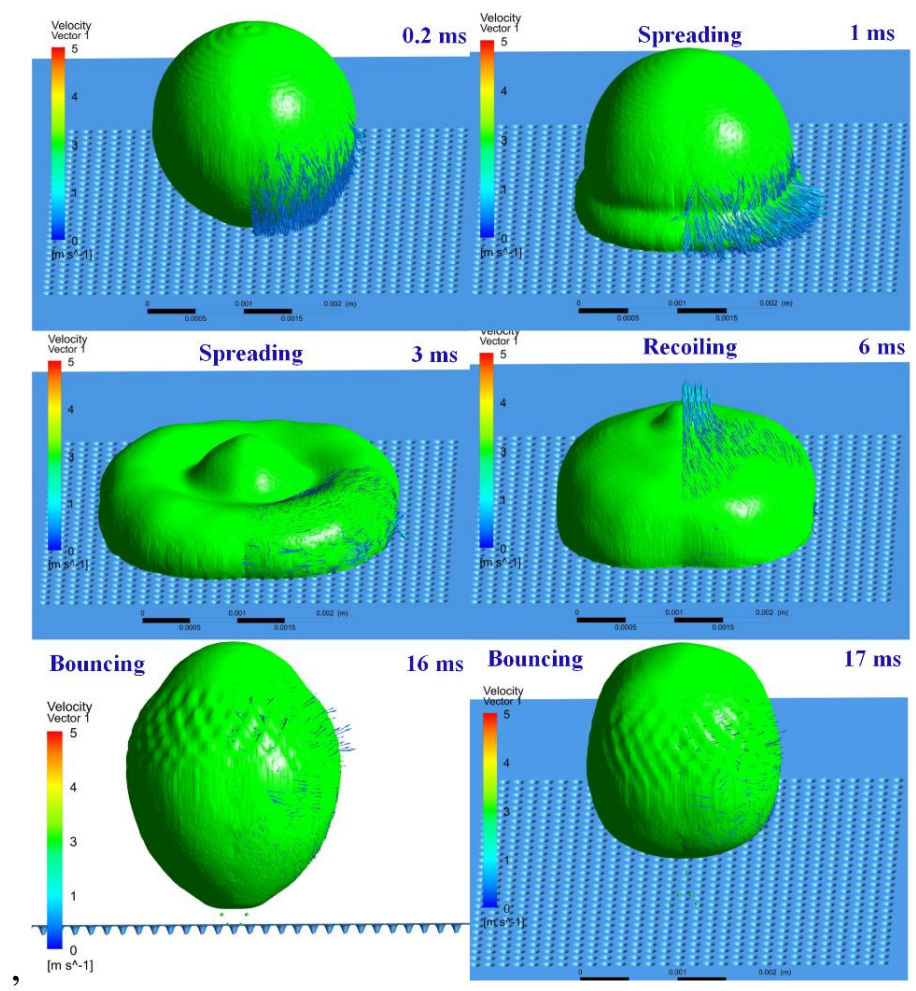

(a) 

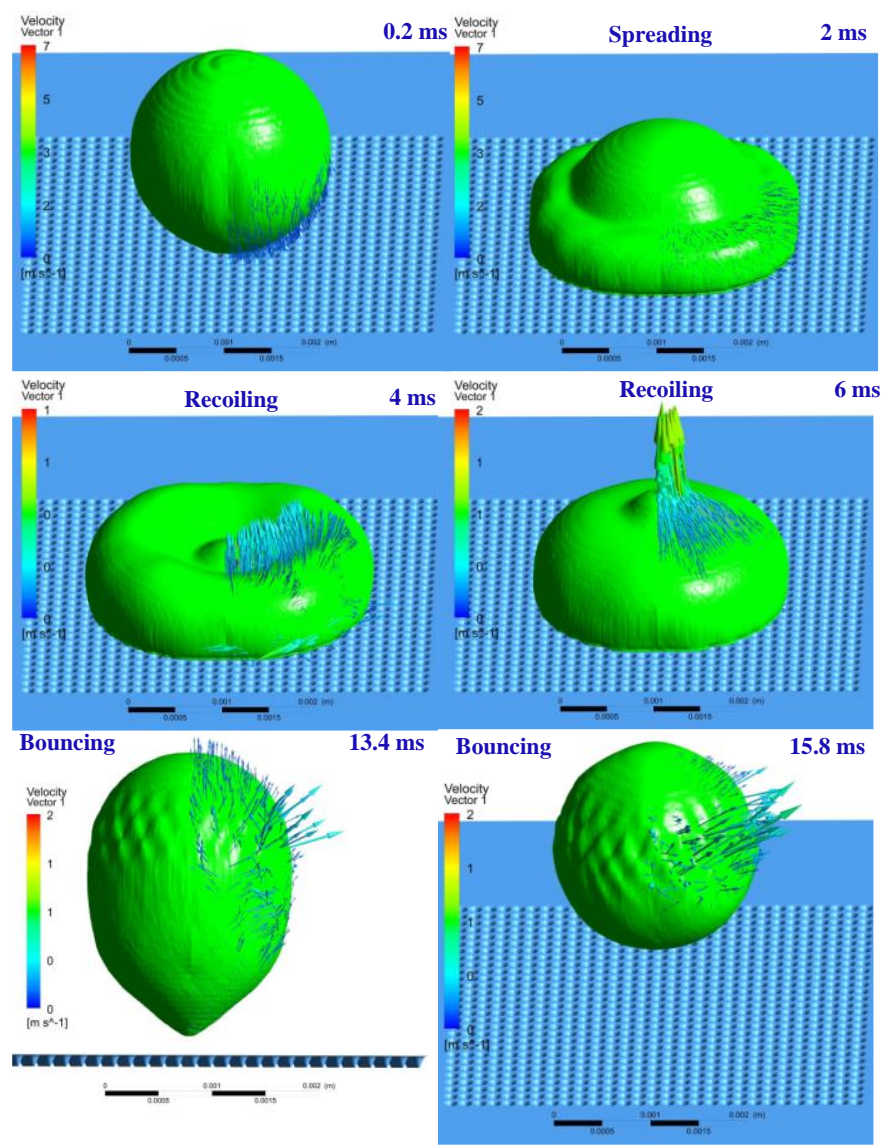

(b)
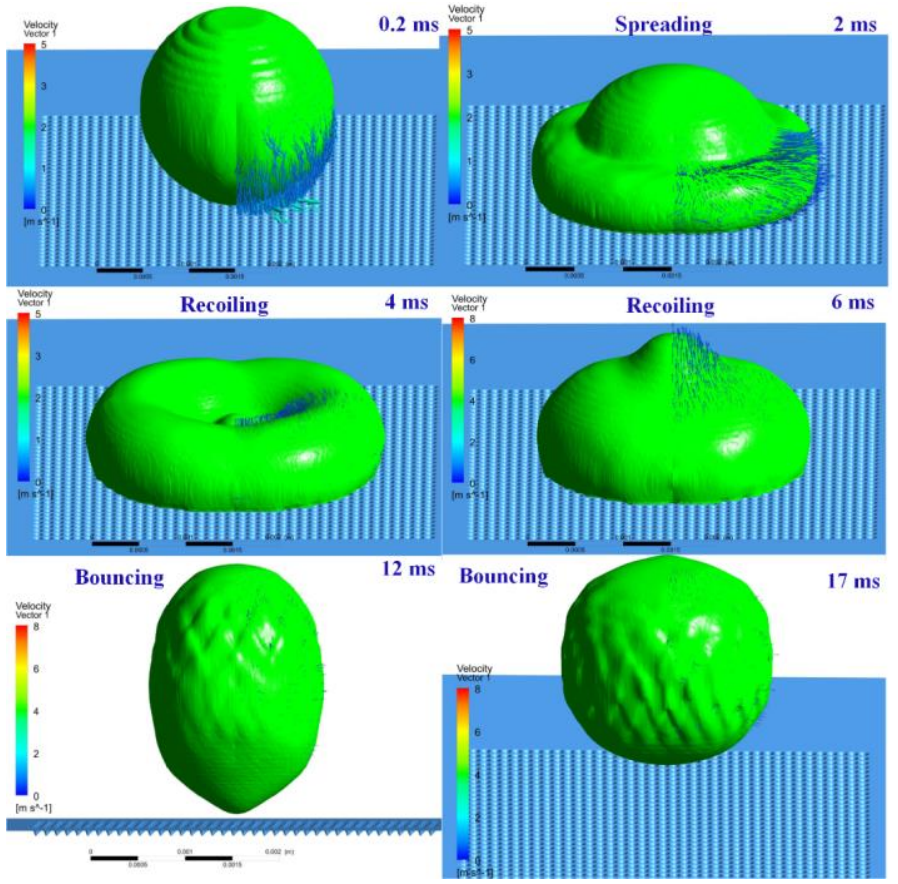

(c)

Fig. 10 Time evolution of water drops with impacting velocity $0.5 \mathrm{~m} / \mathrm{s}$ at (a) P150 (revised; We $=7.26$, Case 5), (b) P150 (optimised; $W e=7.26$, Case 6) and (c) P110 (optimised; $W e=7.26$, Case 7). 
Fig. 11 presents the $D^{*}$ and $h^{*}$ of the droplet for three textured substrates at We 7.26 to quantitatively analyse the water droplet's dynamic behaviour. $D *$ can reflect the water droplet's spreading and compressing during the impacting process. As shown in Fig. 11 (a), the water droplet on the P150 (revised) substrate has the maximum value of $D^{*}(1.49)$ at $3 \mathrm{~ms}$, which means the water droplet has the maximum spreading diameter at that moment. However, the P150 (optimised) and P110 (optimised) substrates have a smaller peak value of $D^{*}$ compared with the P150 (revised), which are 1.46 and 1.38, respectively. In the recoiling stage, the water droplet starts retracting from the moment of possessing maximum $D^{*}$ until detachment from the surface and entering the rebound stage. Fig. 11 (a) shows that P110 (optimised) has the shortest recoiling stage, which means the superhydrophobic surface has less adhesion force; hence, the water droplet can retract quickly. As shown in Fig. 11 (b), $h^{*}$ was displayed to reflect the water droplet's rebound behaviour in the impacting process. In the rebound stage, the $h^{*}$ value shows a steady increasing trend until the water droplet reaches the highest point. Thus, the point with the maximum $h^{*}$ value represents the end of the rising stage. The water droplet on P150 (revised) substrate has an extremely short (less than $1 \mathrm{~ms}$ ) rising stage. However, the rebound time for the P150 (optimised) and P110 (optimised) is approximately $11 \mathrm{~ms}$. Thus, the simulation results proved that water droplets on the superhydrophobic substrates have shorter recoiling time and longer rebounding time than on the hydrophobic substrate.

The rebound stage's starting point can also be reflected in the variations of the air pockets' average pressure at We 7.26, as shown in Fig. 11 (c). The average pressure of the centre Gaussian hole in Fig. 2 (b) was employed to represent the air pressure pocket due to the dynamic impacting process resulting in a varied area of the substrate being affected by the water droplet. The average air pressure is about zero (ambient pressure) due to the water droplet detachment from the surface during the rebound stage. 


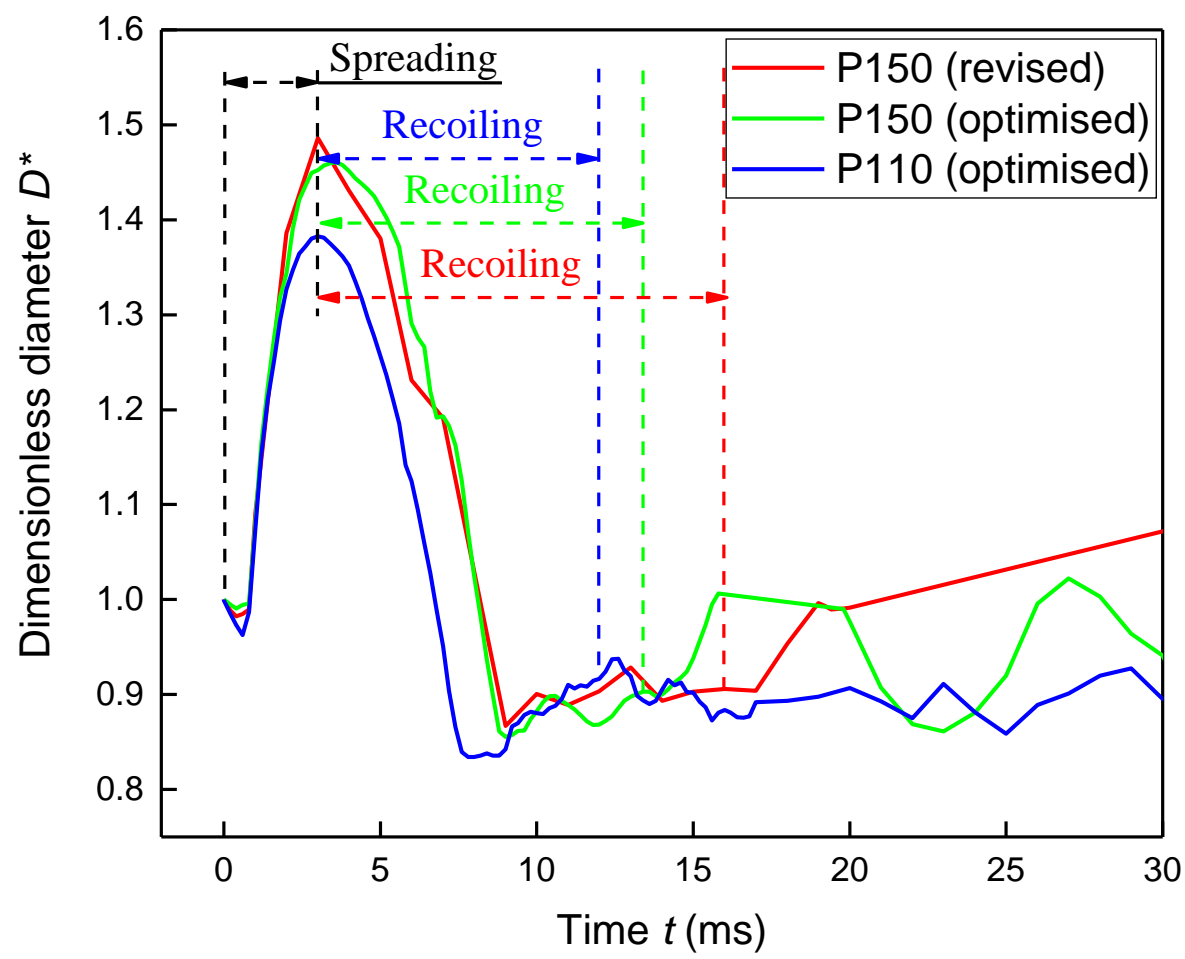

(a)

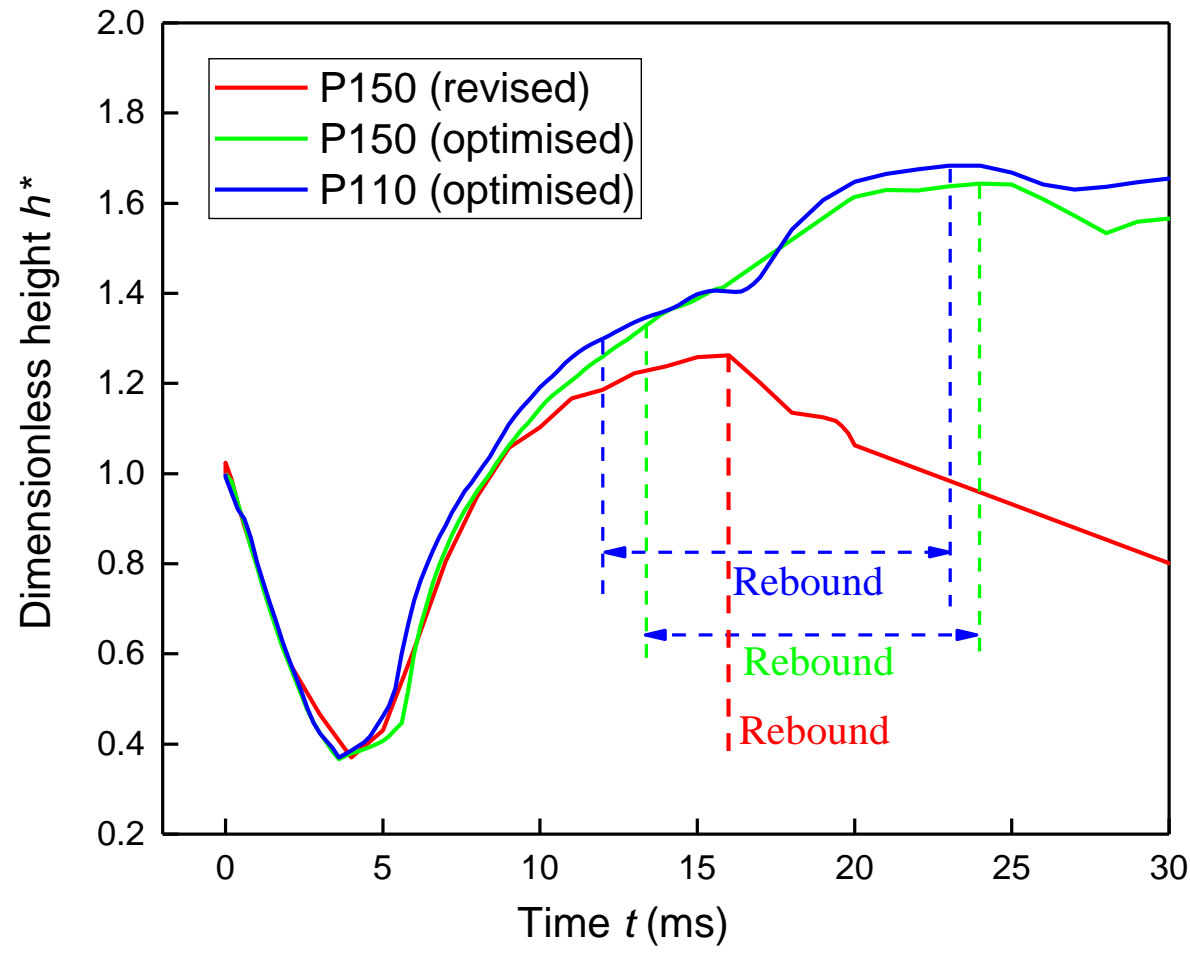

(b) 


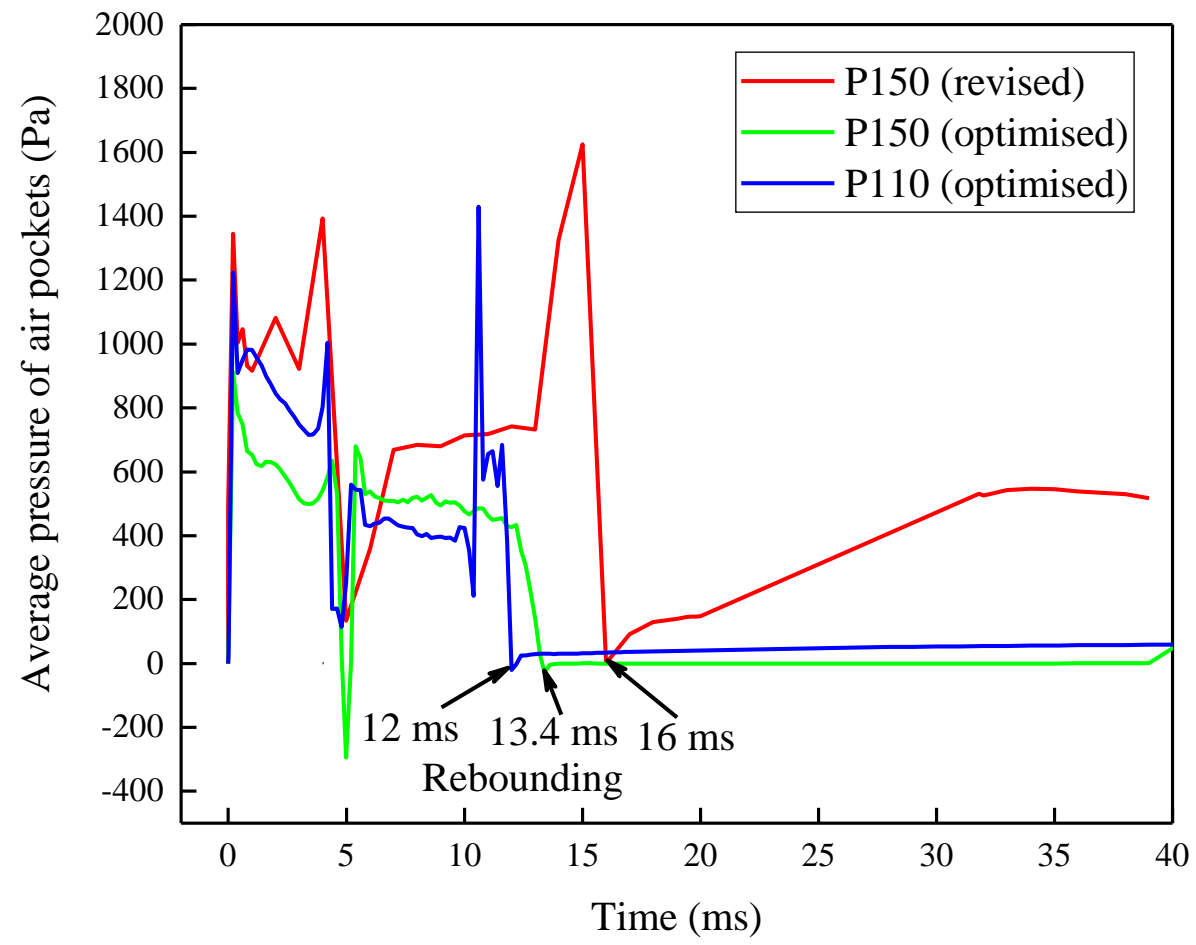

(c)

Fig. 11 Time evolution of (a) $D^{*}$, (b) $h^{*}$ and (c) average pressure of air pocket (We 7.26).

\section{Experimental validation}

Experiments were carried out to validate the simulated contact angles. Specimens (referred Table 2) were prepared by nanosecond pulsed laser machining process. These specimens were processed in a vacuum oven using silane reagents $(1 \mathrm{H}, 1 \mathrm{H}, 2 \mathrm{H}, 2 \mathrm{H}-$ Perfluorooctyltriethoxysilane, 97\%, Alfa Aesar Ltd) at $100{ }^{\circ} \mathrm{C}$ for 12 hours to reduce their surface free energies. The laser ablation parameters are listed in Table 4.

Table 4 Laser ablation parameters

\begin{tabular}{lllll}
\hline Laser power $(\mathrm{W})$ & Pitch $(\mu \mathrm{m})$ & $\begin{array}{l}\text { Pulse repetition } \\
\text { rate }(\mathrm{kHz})\end{array}$ & $\begin{array}{l}\text { Feed rate } \\
(\mathrm{mm} / \mathrm{min})\end{array}$ & $\begin{array}{l}\text { Duration time } \\
(\mathrm{s})\end{array}$ \\
\hline $4-14$ & 110,150 & 100 & 200 & 0.4 \\
\hline
\end{tabular}

Surface topography was measured by an optical 3D surface measurement device (i.e. Alicona G4), while the contact angle was measured by a drop shape analyser DSA25B (Kruss Ltd.) in a set-up shown in Fig. 12 (a) after the silanization process. The selected water droplet volume was $5 \mu \mathrm{L}$. For each specimen, the apparent contact angle of the water droplet was measured 
three times and the average value was adopted. A high-speed camera (Phantom v2012) (shown in Fig. 12 (b)) was used to capture images of the dynamic impacting process of water droplets on smooth and superhydrophobic substrates. The frame rate was set at $1000 \mathrm{fps}$ in this test, the water droplets were released from certain distances as shown in Table 5 to the surface to achieve contact impacting velocities of $0.5 \mathrm{~m} / \mathrm{s}$ and $1 \mathrm{~m} / \mathrm{s}$ respectively.

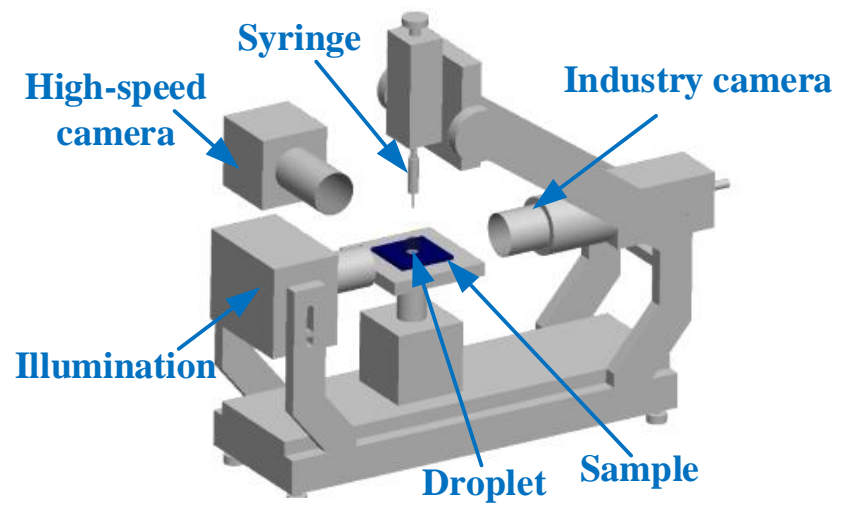

(a)

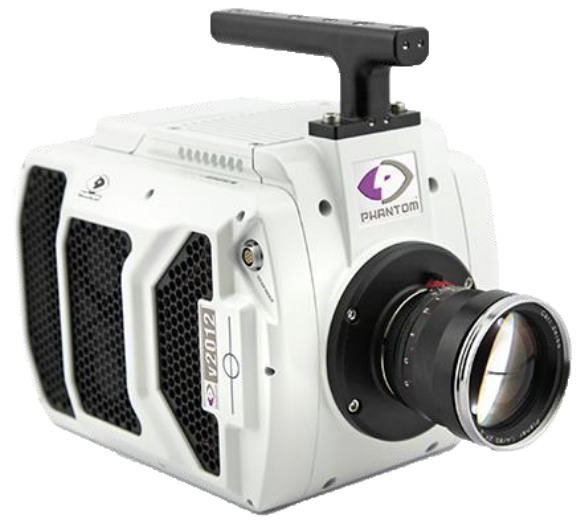

(b)

Fig. 12 Phantom v2012 high-speed camera.

Table 5 Conditions of high-speed camera test.

\begin{tabular}{llll}
\hline $\begin{array}{l}\text { Volume of water } \\
\text { droplet }(\mu \mathrm{L})\end{array}$ & Distance $(\mathrm{mm})$ & $\begin{array}{l}\text { Impacting velocity } \\
(\mathrm{m} / \mathrm{s})\end{array}$ & Weber number \\
\hline 5 & 12.7 & 0.5 & 7.26 \\
\hline 5 & 51 & 1 & 29 \\
\hline
\end{tabular}

\subsection{Static contact angle and profiles}

Fig. 13 show comparisons between the experimental and designed profile of microstructures and 3D morphologies of laser ablated specimens. In all specimens, the specimen P150-14 W has a most similar profile with the designed specimen P150 (revised). The specimen P110-14 $\mathrm{W}$ has a most similar profile with the designed specimen P110 (optimised). However, not all the designed profile can be obtained in the experiments. There are no specimens have similar dimensions with P150 (optimised).

For specimens P150 (revised) and P110 (optimized), the actual width of Gaussian hole is smaller than the optimised value $6 c$, which results in an increased fraction of solid-liquid contact area $(f)$. Consequently, the actual contact angle is smaller than the predicted value, as shown in Fig. 14. 


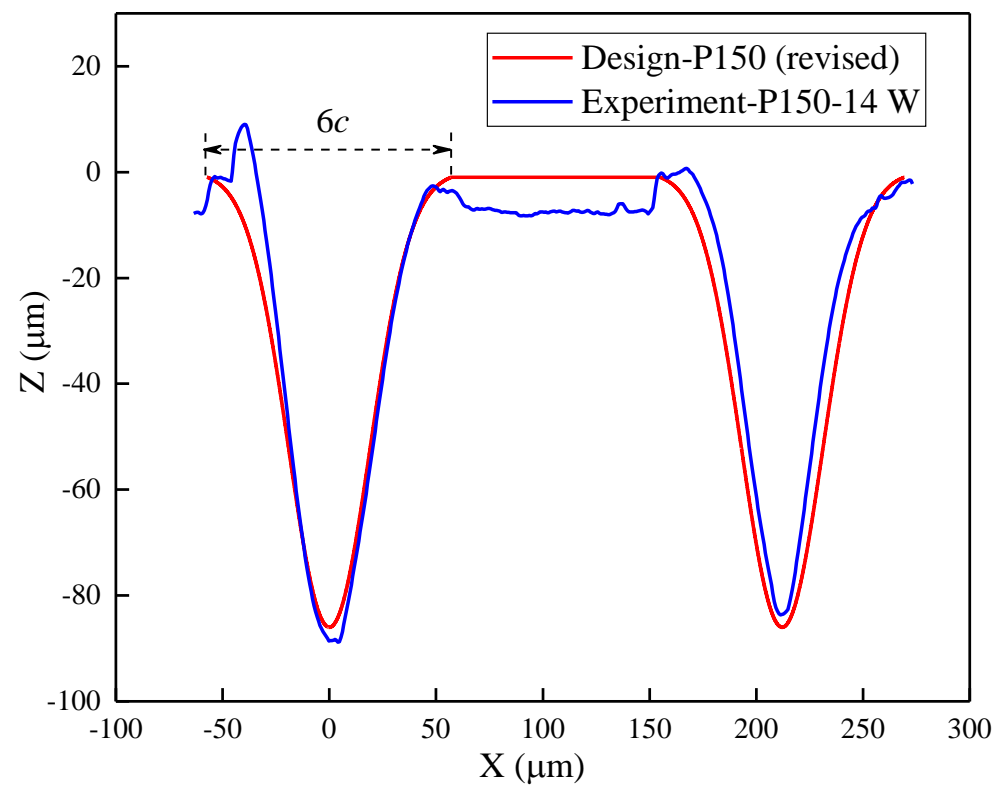

(a)
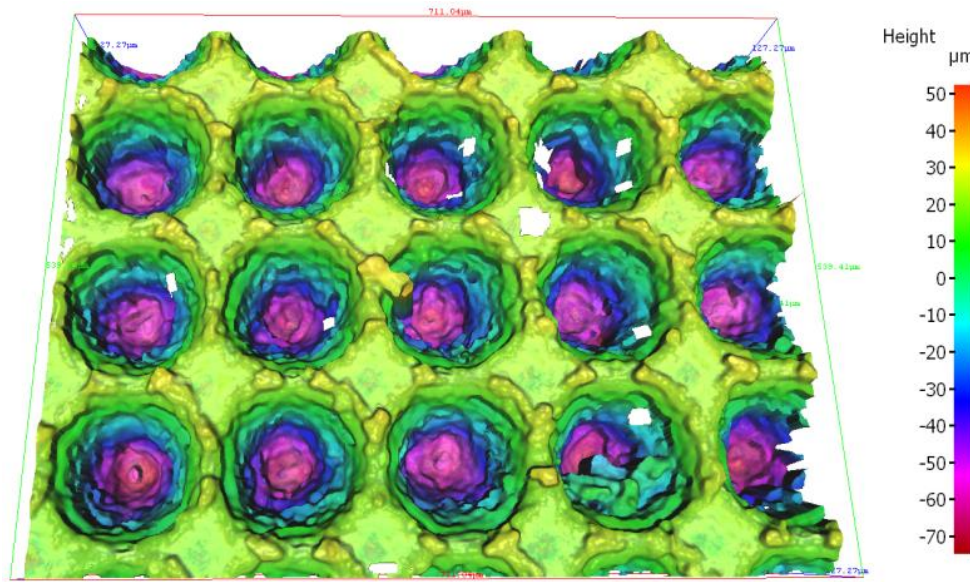

(b)

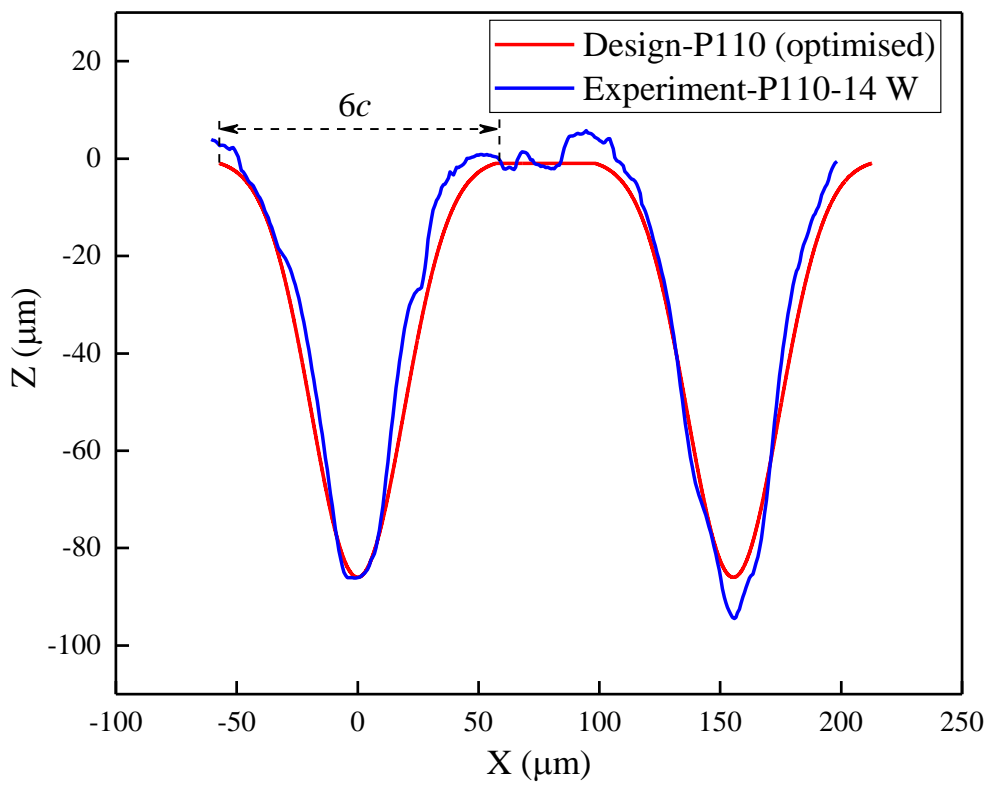

(c) 

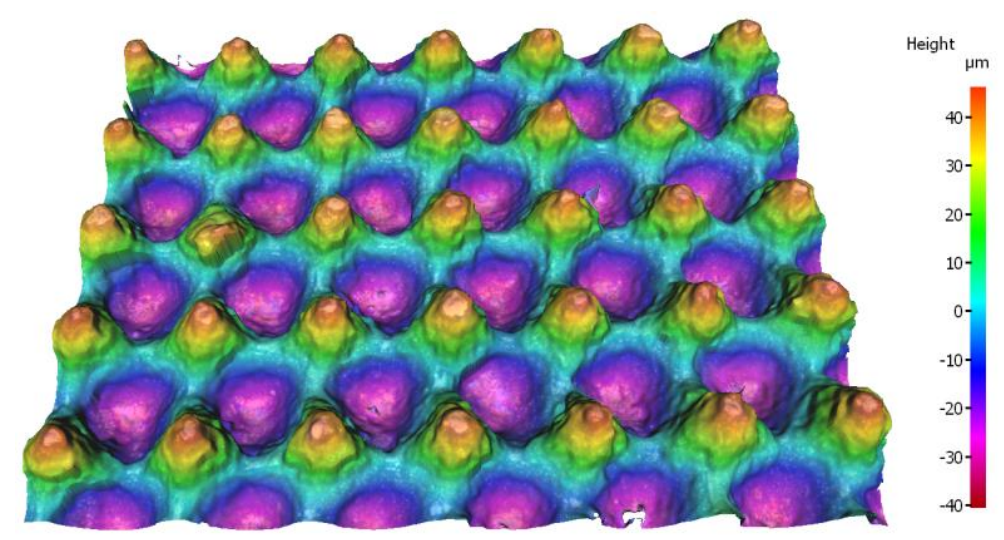

(d)

Fig. 13 Comparison of design profile and experiments for specimens with pitches of (a) 150 $\mu \mathrm{m}$ and (c) $110 \mu \mathrm{m}$; (b) and (d): 3D morphologies of P150-14 W and P110-14W.

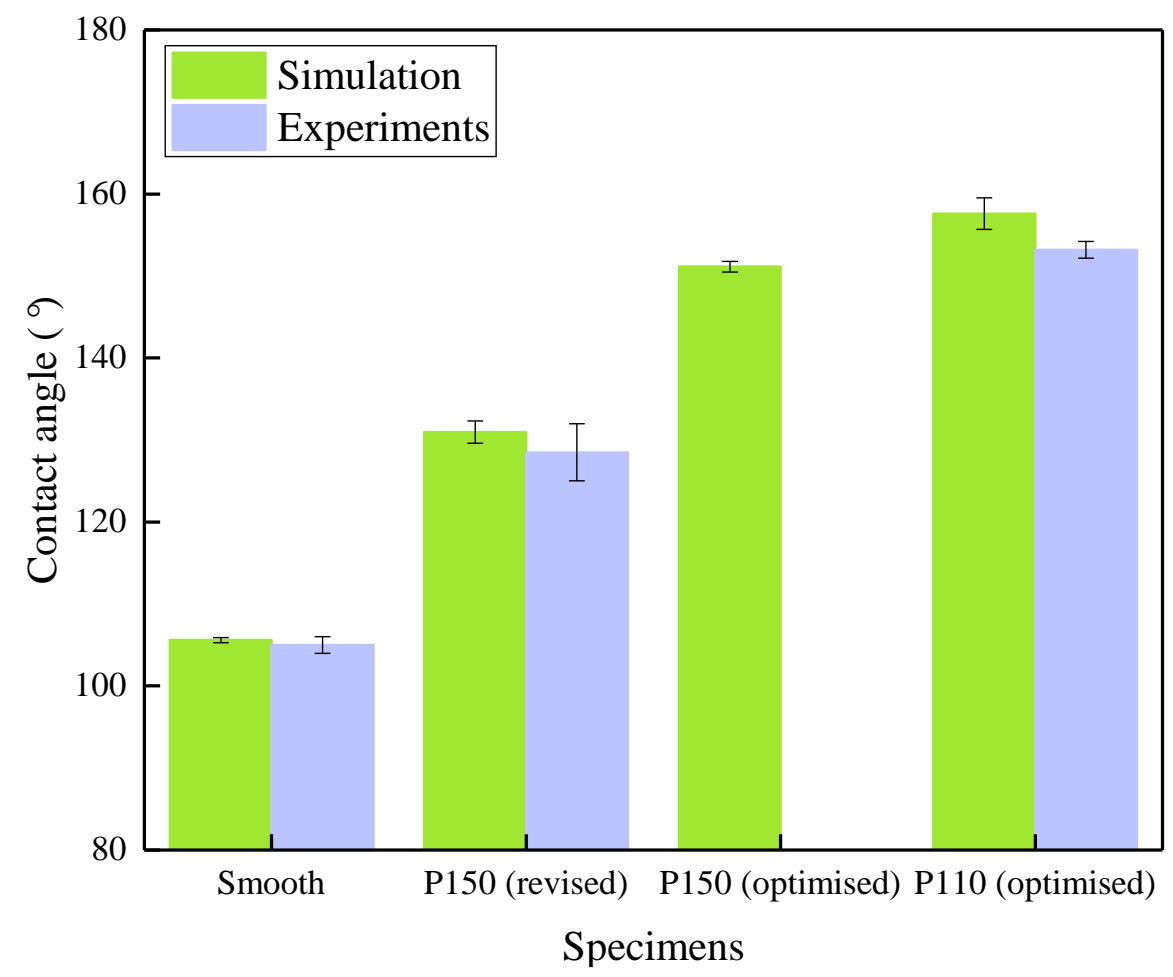

Fig. 14 Comparison between the simulated and measured contact angles.

Fig. 15 shows the captured image of the droplets in the contact angle measurements. For P11014W specimen (i.e. P110 (optimized)), light passes below the droplet, indicating that air pockets exist, so that the droplet is in the Cassie-Baxter state. 


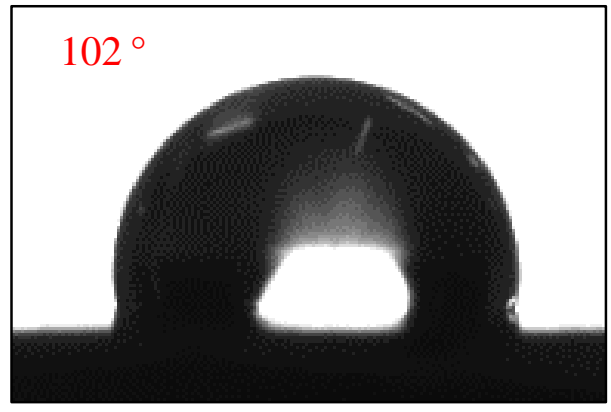

(a) Smooth

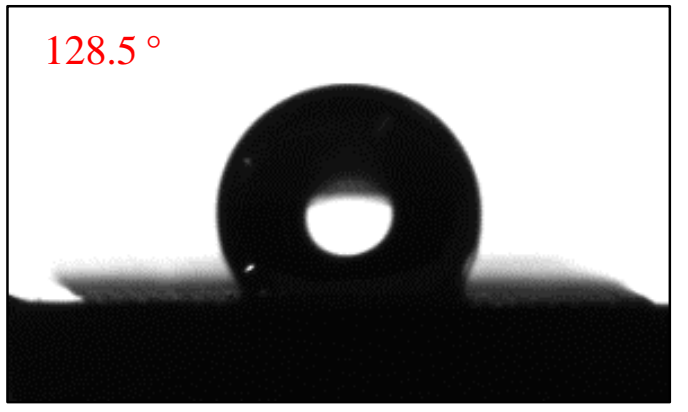

(b) P150-14W

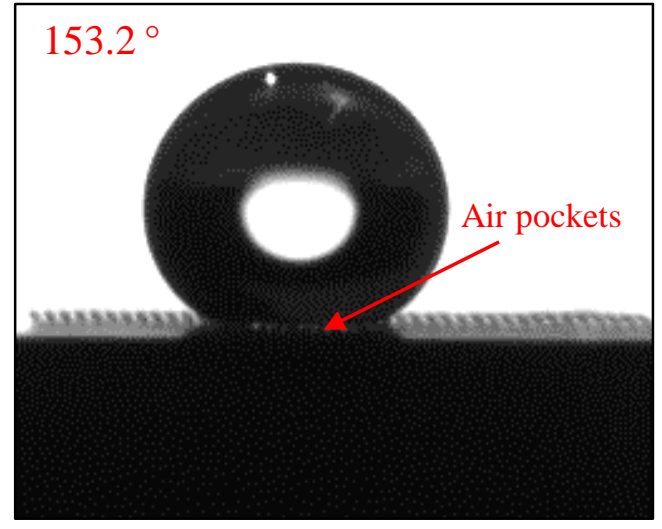

(c) $\mathrm{P} 110-14 \mathrm{~W}$

Fig. 15 Water droplet shapes for different specimens.

\subsection{High-speed camera test results}

The sequential images of a simulated water droplet with a Weber number of 7.26 impacting on the P150 (revised) specimen were compared with the test results of a high-speed camera on specimen P150-14 W. As presented in Fig. 16, the shapes of the simulated water droplet are in good agreement with the high-speed camera observation results from 0 to $6 \mathrm{~ms}$. This is because that the water droplet is in the spreading and initial recoiling stages between 0 and $6 \mathrm{~ms}$, and the inertia of water droplet dominated the motion. However, unlike the simulation results, the water droplet in the experiment did not detach from the substrate at $16 \mathrm{~ms}$. The actual laser ablated specimen is rougher than the $3 \mathrm{D}$ geometry model due to the existing slags and recast layers on the surface, as shown in Fig. 13 (a), which results in larger solid-liquid contact area than the simulation. In the impacting process, the kinetic energy was gradually dissipated by the adhesion force between the solid and liquid and by the viscous force during the recoiling stage. Hence, more kinetic energy is dissipated in the recoiling process and there is insufficient kinetic energy to raise the water droplet from the substrate in the experiments. 


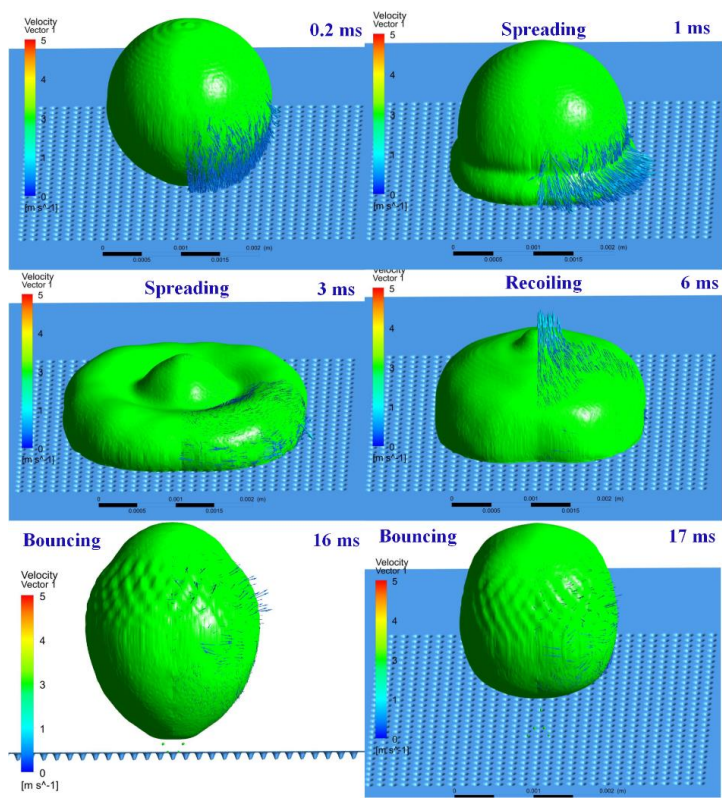

(a)

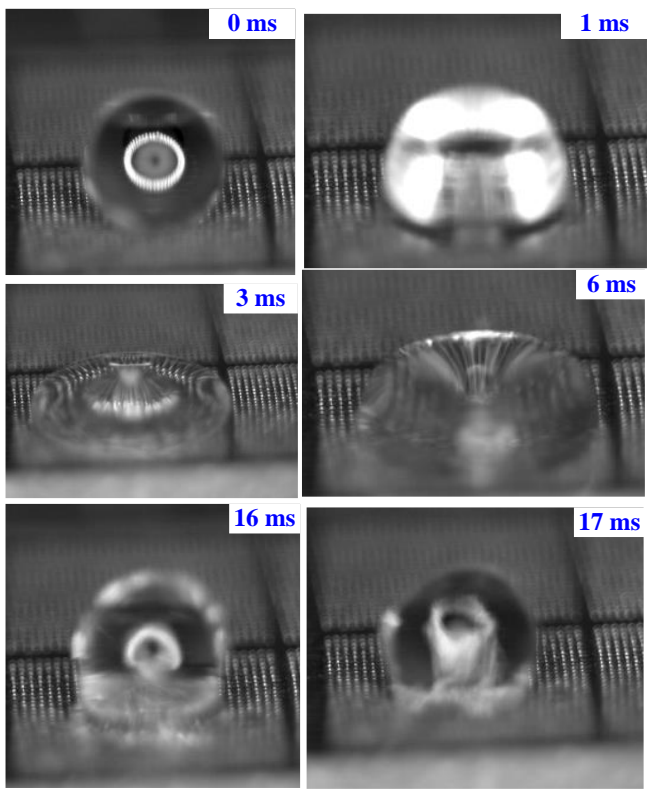

(b)

Fig. 16 Time evolution of water drops with impacting velocity $0.5 \mathrm{~m} / \mathrm{s}$ : (a) simulation results of P150 (revised) and (b) captured images of P150-14 W.

Similarly, the simulated and experimental images of a water droplet with a Weber number of 7.26 impacting the P110 (optimised) specimen are presented in Fig. 17. The results also show good agreement in the spreading and recoiling stages. Based on the simulation results (Fig. 17 (a)), it can know that the water droplet would rebound from the substrate at $12 \mathrm{~ms}$. However, the high-speed camera experiment shows that the time of detachment is $16 \mathrm{~ms}$. This minor deviation (i.e. $4 \mathrm{~ms}$ difference of detachment time) also resulted from the rougher morphology of laser ablated specimen than the ideal geometry model. Furthermore, water droplet has horizontal momentum when bounces upwards at $16 \mathrm{~ms}$ in the experiments. Unlike that observed in VOF simulation, the shape of water droplet has the trend towards the left side and is not symmetrical as a result of some imperfections (e.g. debris, recast layer) on the laser ablated surface. In addition, after the water droplet detached from the substrate, both the simulated and the experimental results indicate that the water droplet shows a secondary oscillation in the air. At 16-19 ms in the experiment, the bottom region of the droplet continues to move upward under the influence of inertia and surface tension. The bottom surface has a higher upward velocity than top surface, which results in oscillation of water droplet. Similar behaviour can be found in simulation between 12 and $17 \mathrm{~ms}$ also as shown in Fig 17 (a). 


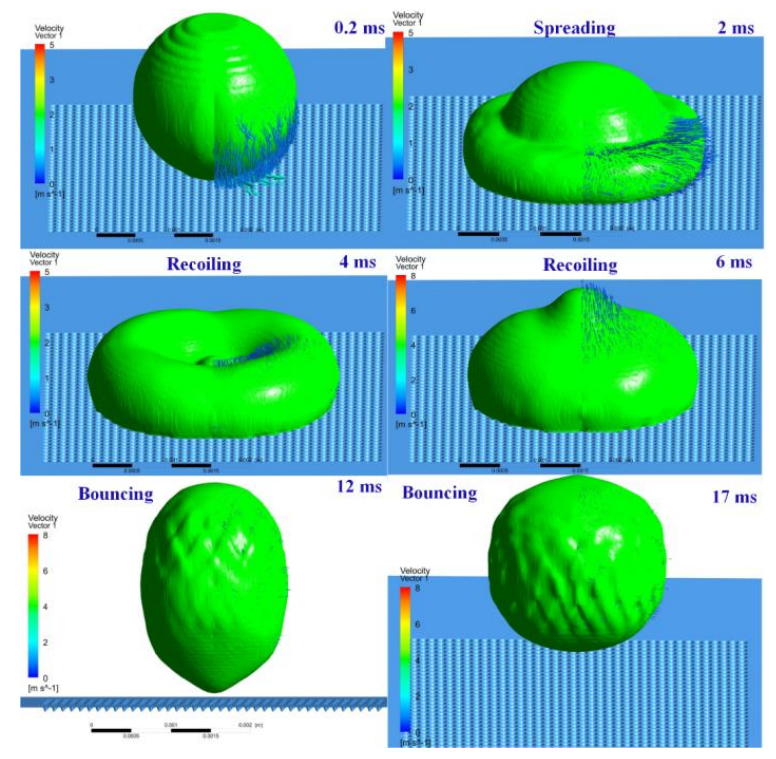

(a)

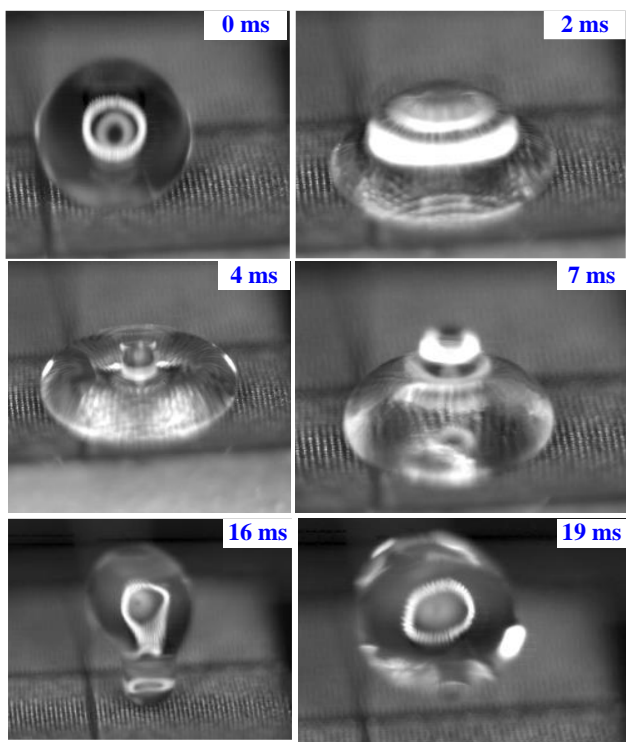

(b)

Fig. 17 Time evolution of water drops with impacting velocity $0.5 \mathrm{~m} / \mathrm{s}$ : (a) simulation results of P110 (optimised) and (b) captured images of P110-14 W.

To further investigate the adhesion capability of a water droplet on the superhydrophobic substrate, a higher impacting velocity of $1 \mathrm{~m} / \mathrm{s}(W e=29)$ was conducted on the specimen P150$14 \mathrm{~W}$ (hydrophobic substrate) and the specimen P110-14 W (superhydrophobic substrate). As shown in Fig. 18, the water droplet did not detach from the specimen P150-14 W; however, the droplet rebounded from the specimen P110-14 W at $35 \mathrm{~ms}$ and $75 \mathrm{~ms}$. Therefore, the laser ablated superhydrophobic surface has a shorter contact time with water droplet than the hydrophobic substrate. It also means the laser ablated superhydrophobic surface has a lower adhesion force to the water droplet than the hydrophobic surface. The low adhesion force to the water droplet will help superhydrophobic surface to be used in practical applications. For instance, the corrosion resistance was remarkably improved by the low water adhesion of the substrate [36]; the decreasing adhesion on superhydrophobic surfaces help drag reduction, and the greatest drag-reduction ratio is $30 \%-40 \%$ at low adhesion [8]. 


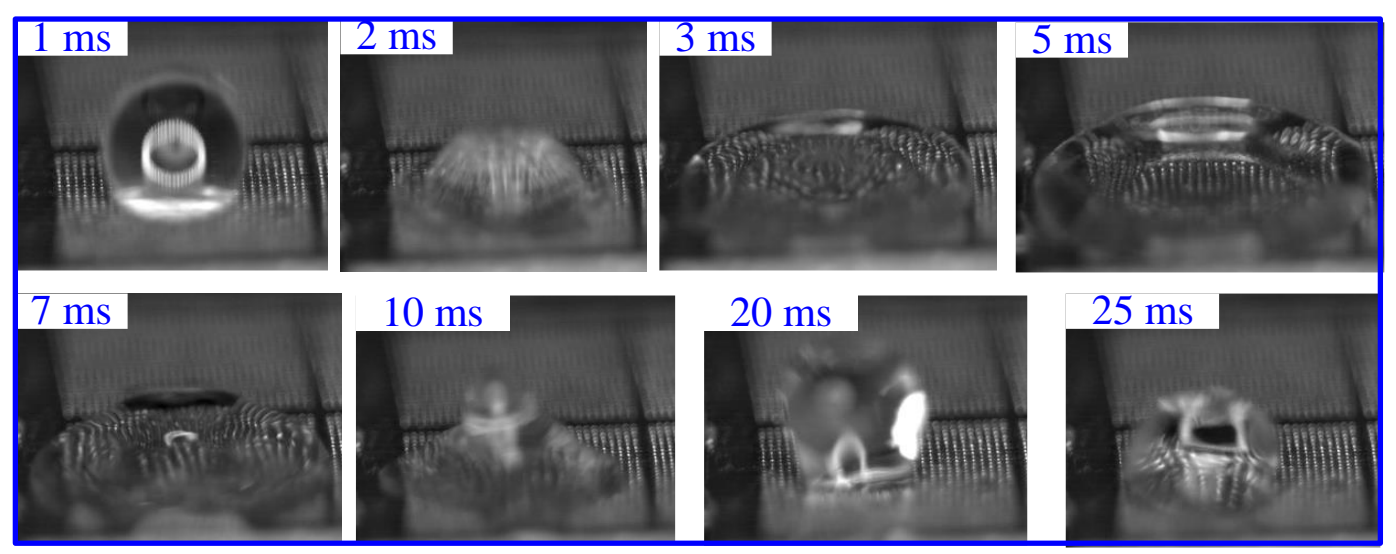

(a)

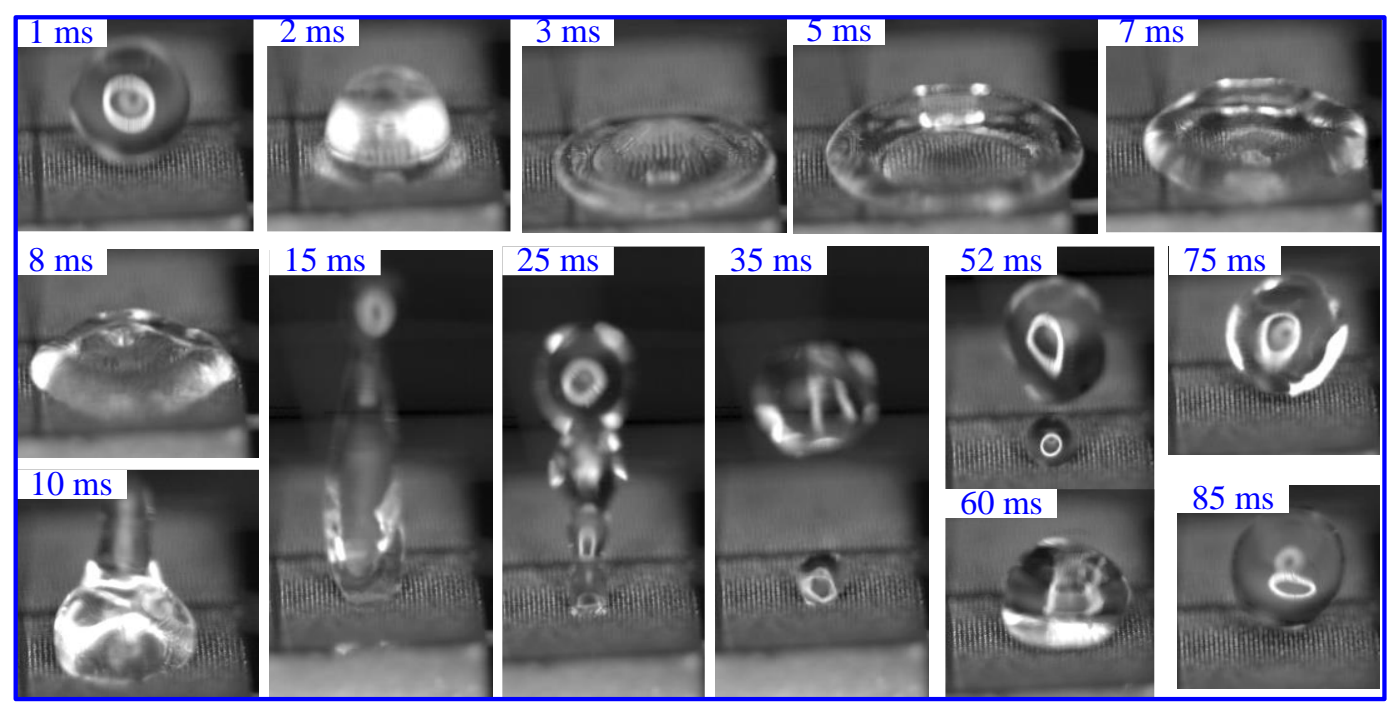

(b)

Fig. 18 High-speed camera captured images under impacting velocity $1 \mathrm{~m} / \mathrm{s}$ of (a) P150-14 W and (b) P110-14 W.

The above results of measurement of static contact angle and high speed camera test proved the effectiveness of VOF simulation at Weber number of 0.026 and 7.26 to study hydrophobicity of different microstructures. VOF simulations can provide detailed information about the interaction among gas, liquid and solid phases, such as contact angle, air pocket's pressure, kinetic and potential energy of water droplet. The static contact angle test proved that VOF simulation can be used as a design-assisted tool to predict the contact angle of different designed structures. Thus, VOF simulation can capture the key features of water droplet during dynamic impacting process with real length and time scales, i.e. millimetre level and ms level although the geometry model does not consider the micro/nano asperities. 


\section{Conclusions}

In this study, CFD simulation was conducted to predict the hydrophobicity of different substrates and to explore the underlying mechanism of different wetting states. Moreover, the laser ablation experiments and high-speed camera test were conducted to validate the simulation results. The conclusions are as follows:

1. In a quasi-static impacting process $(W e=0.026)$, the air pockets' pressure shows a declining trend for hydrophobic substrate P150 (revised). However, the superhydrophobic substrates P150 (optimised) and P110 (optimised) trapped a large volume of air with high pressure resulting in a stable intermediate state and a Cassie-Baxter state, respectively.

2. The superhydrophobic substrate has the maximum potential energy and kinetic energy, which helps explain its low adhesion and self-cleaning properties.

3. In the impacting process at a Weber number of 7.26, with the increase of the three specimens' hydrophobicity, the water droplet detachment occurs earlier, varying from $16 \mathrm{~ms}$ to $12 \mathrm{~ms}$. Superhydrophobicity results in smaller dimensionless diameter, larger dimensionless height, shorter recoiling time and longer rebound time than the hydrophobic substrate.

4. The simulated and measured contact angles have minor deviations from the experimental value, as the machined specimens P150-14 W and P110-14 W have similar dimensions with the designed substrates P150 (revised)) and P110 (optimised)) respectively.

5. The dynamic impacting behaviour of a water droplet at a Weber number of 7.26 and 29 proved that the laser ablated superhydrophobic surface has a lower adhesion force to the water droplet than hydrophobic surface.

\section{Acknowledgements}

This research was undertaken in the context of MICROMAN project ("Process Fingerprint for Zero-defect Net-shape MICROMANufacturing", http://www.microman.mek.dtu.dk/). MICROMAN is a European Training Network supported by Horizon 2020, the EU Framework Programme for Research and Innovation (Project ID: 674801). The authors would also gratefully acknowledge the financial support from the EPSRC (EP/K018345/1) and Royal society-NSFC International exchange scheme (IE141422 and IECINSFC 1181474) for this research. The authors also acknowledge the use of the EPSRC (EP/K000586/1) funded ARCHIE-WeSt High-Performance Computer at the University of Strathclyde. 


\section{Data Statement}

All data underpinning this publication are openly available from the University of Strathclyde KnowledgeBase at https://doi.org/10.15129/32d18bb9-884b-4416-92c1-1b5919a5869c.

\section{References}

[1] Khojasteh D, Kazerooni M, Salarian S, Kamali R. Droplet impact on superhydrophobic surfaces: A review of recent developments. J Ind Eng Chem 2016;42:1-14. doi:10.1016/j.jiec.2016.07.027.

[2] Feng L, Li S, Li Y, Li H, Zhang L, Zhai J, et al. Super-hydrophobic surfaces: from natural to artificial. Adv Mater 2002;14:1857-60. doi:10.1002/adma.200290020.

[3] Bachus KJ, Mats L, Choi HW, Gibson GTT, Oleschuk RD. Fabrication of Patterned Superhydrophobic/Hydrophilic Substrates by Laser Micromachining for Small Volume Deposition and Droplet-Based Fluorescence. ACS Appl Mater Interfaces 2017;9:7629_ 36. doi:10.1021/acsami.6b16363.

[4] Salehi M, Mozammel M, Emarati SM. Superhydrophobic and corrosion resistant properties of electrodeposited Ni-TiO 2 /TMPSi nanocomposite coating. Colloids Surfaces A Physicochem Ang Asp 2019;573:196-204. doi:10.1016/j.colsurfa.2019.04.024.

[5] Zhang X, Zhao J, Mo J, Sun R, Li Z, Guo Z. Fabrication of superhydrophobic aluminum surface by droplet etching and chemical modification. Colloids Surfaces A Physicochem Eng Asp 2019;567:205-12. doi:10.1016/j.colsurfa.2019.01.046.

[6] Wen G, Huang JX, Guo ZG. Energy-effective superhydrophobic nanocoating based on recycled eggshell. Colloids Surfaces A Physicochem Eng Asp 2019;568:20-8. doi:10.1016/j.colsurfa.2019.01.067.

[7] Moaven K, Rad M, Taeibi-Rahni M. Experimental investigation of viscous drag reduction of superhydrophobic nano-coating in laminar and turbulent flows. Exp Therm Fluid Sci 2013;51:239-43. doi:10.1016/j.expthermflusci.2013.08.003.

[8] Zhang H, Yin L, Li L, Shi S, Wang Y, Liu X. Wettability and drag reduction of a superhydrophobic aluminum surface. RSC Adv 2016;6:14034-41. doi:10.1039/c5ra23842k. 
[9] Daniello RJ, Waterhouse NE, Rothstein JP. Drag reduction in turbulent flows over superhydrophobic surfaces. Phys Fluids 2009;21. doi:10.1063/1.3207885.

[10] Yuan S, Strobbe D, Kruth JP, Van Puyvelde P, Van Der Bruggen B. Super-hydrophobic 3D printed polysulfone membranes with a switchable wettability by self-assembled candle soot for efficient gravity-driven oil/water separation. J Mater Chem A 2017;5:25401-9. doi:10.1039/c7ta08836a.

[11] Liu Z, Zhang F, Chen Y, Zhang H, Han Y, Liu J, et al. Electrochemical fabrication of superhydrophobic passive films on aeronautic steel surface. Colloids Surfaces A Physicochem Eng Asp 2019;572:317-25. doi:10.1016/j.colsurfa.2019.04.033.

[12] Kinoshita H, Ogasahara A, Fukuda Y, Ohmae N. Superhydrophobic/superhydrophilic micropatterning on a carbon nanotube film using a laser plasma-type hyperthermal atom beam facility. Carbon N Y 2010;48:4403-8. doi:10.1016/j.carbon.2010.07.056.

[13] Lau KKS, Bico J, Teo KBK, Chhowalla M, Amaratunga GAJ, Milne WI, et al. Superhydrophobic Carbon Nanotube Forests. Nano Lett 2003;3:1701-5. doi:10.1021/n1034704t.

[14] Latthe SS, Imai H, Ganesan V, Rao AV. Superhydrophobic silica films by sol-gel coprecursor method. Appl Surf Sci 2009;256:217-22. doi:10.1016/j.apsusc.2009.07.113.

[15] Gleason KK, Rutledge GC, Gupta M, Ma M, Mao Y. Superhydrophobic fibers produced by electrospinning and chemical vapor deposition. US Pat Appl Publ 2007:26pp.

[16] Zhang X, Guo Y, Zhang P, Wu Z, Zhang Z. Superhydrophobic CuO@Cu2S nanoplate vertical arrays on copper surfaces. Mater Lett 2010;64:1200-3. doi:10.1016/j.matlet.2010.02.050.

[17] Pan G, Xiao X, Ye Z. Fabrication of stable superhydrophobic coating on fabric with mechanical durability, UV resistance and high oil-water separation efficiency. Surf Coatings Technol 2019;360:318-28. doi:10.1016/j.surfcoat.2018.12.094.

[18] Liu C, Zhan H, Yu J, Liu R, Zhang Q, Liu Y, et al. Design of superhydrophobic pillars with robustness. Surf Coatings Technol 2019;361:342-8. doi:10.1016/j.surfcoat.2019.01.041.

[19] Mumm F, Helvoort ATJ Van, Sikorski P. Easy Route to Superhydrophobic. ACS Nano 2009;3:2647-52. 
[20] Fürstner R, Barthlott W, Neinhuis C, Walzel P. Wetting and self-cleaning properties of artificial superhydrophobic surfaces. Langmuir 2005;21:956-61. doi:10.1021/la0401011.

[21] Rights M. Hydrophobicity of pyramid structures fabricated by micro milling . In : 2017 World Congress on Micro and Nano Manufacturing , 2017-03-27 - This version is available at https://strathprints.strath.ac.uk/60698/. Stud Comput Intell 2017;7:2-6.

[22] Cai Y, Chang W, Luo X, Sousa AML, Lau KHA, Qin Y. Superhydrophobic structures on 316L stainless steel surfaces machined by nanosecond pulsed laser. Precis Eng 2018;52:266-75. doi:10.1016/j.precisioneng.2018.01.004.

[23] Volkov RS, Strizhak PA, Misyura SY, Lezhnin SI, Morozov VS. The influence of key factors on the heat and mass transfer of a sessile droplet. Exp Therm Fluid Sci 2018;99:59-70. doi:10.1016/j.expthermflusci.2018.07.010.

[24] Kulju S, Riegger L, Koltay P, Mattila K, Hyväluoma J. Fluid flow simulations meet high-speed video: Computer vision comparison of droplet dynamics. J Colloid Interface Sci 2018;522:48-56. doi:10.1016/j.jcis.2018.03.053.

[25] Yuan WZ, Zhang LZ. Lattice Boltzmann simulation of droplets impacting on superhydrophobic surfaces with randomly distributed rough structures. Langmuir 2017;33:820-9. doi:10.1021/acs.langmuir.6b04041.

[26] Ke Z, Shi J, Zhang B, Chen CL. Numerical investigation of condensation on microstructured surface with wettability patterns. Int $\mathbf{J}$ Heat Mass Transf 2017;115:1161-72. doi:10.1016/j.ijheatmasstransfer.2017.08.121.

[27] Tembely M, Attarzadeh R, Dolatabadi A. On the numerical modeling of supercooled micro-droplet impact and freezing on superhydrophobic surfaces. Int J Heat Mass Transf 2018;127:193-202. doi:10.1016/j.ijheatmasstransfer.2018.06.104.

[28] Liu C, Zhu L, Bu W, Liang Y. Superhydrophobic surfaces: from nature to biomimetic through VOF simulation. Micron 2018;107:94-100. doi:10.1016/j.micron.2018.01.013.

[29] Yun S. Bouncing of an ellipsoidal drop on a superhydrophobic surface. Sci Rep 2017;7:1-9. doi:10.1038/s41598-017-18017-2.

[30] Quan Y, Zhang LZ. Numerical and analytical study of the impinging and bouncing phenomena of droplets on superhydrophobic surfaces with microtextured structures. 
Langmuir 2014;30:11640-9. doi:10.1021/la502836p.

[31] Murugadoss K, Dhar P, Das SK. Role and significance of wetting pressures during droplet impact on structured superhydrophobic surfaces. Eur Phys J E 2017;40:1-10. doi:10.1140/epje/i2017-11491-x.

[32] Zhang W, Zhang R run, Jiang C gang, Wu C wei. Effect of pillar height on the wettability of micro-textured surface: Volume-of-fluid simulations. Int J Adhes Adhes 2017;74:649. doi:10.1016/j.ijadhadh.2016.12.011.

[33] Overview and limitations of the VOF model. Fluent Inc 2006. http://cdlab2.fluid.tuwien.ac.at/LEHRE/TURB/Fluent.Inc/fluent6.3.26/help/html/ug/no de881.htm.

[34] Ursell F. An introduction to fluid dynamis. J Fluid Mech 1959;5:493-4. doi:10.1017/S0022112059220333.

[35] Brackbill JU, Kothe DB, Zemach C. A continuum method for modeling surface tension. J Comput Phys 1992;100:335-54. doi:10.1016/0021-9991(92)90240-Y.

[36] Cui M, Shen Y, Tian H, Yang Y, Feng H, Li J. Influence of water adhesion of superhydrophobic surfaces on their anti-corrosive behavior. Surf Coatings Technol 2018;347:38-45. doi:10.1016/j.surfcoat.2018.04.064. 